\title{
Random attractors for stochastic partly dissipative systems
}

\author{
Christian Kuehn, Alexandra Neamţu and Anne Pein®
}

\begin{abstract}
We prove the existence of a global random attractor for a certain class of stochastic partly dissipative systems. These systems consist of a partial and an ordinary differential equation, where both equations are coupled and perturbed by additive white noise. The deterministic counterpart of such systems and their long-time behaviour have already been considered but there is no theory that deals with the stochastic version of partly dissipative systems in their full generality. We also provide several examples for the application of the theory.
\end{abstract}

Mathematics Subject Classification. 60H15, 37H05, 37L55.

Keywords. Random attractor, Partly dissipative systems, Stochastic partial differential equation, Random dynamical system.

\section{Introduction}

In this work, we study classes of stochastic partial differential equations (SPDEs), which are part of the general partly dissipative system

$$
\begin{aligned}
& \mathrm{d} u_{1}=\left(d \Delta u_{1}-h\left(x, u_{1}\right)-f\left(x, u_{1}, u_{2}\right)\right) \mathrm{d} t+B_{1}\left(x, u_{1}, u_{2}\right) \mathrm{d} W_{1}, \\
& \mathrm{~d} u_{2}=\left(-\sigma(x) u_{2}-g\left(x, u_{1}, u_{2}\right)\right) \mathrm{d} t+B_{2}\left(x, u_{1}, u_{2}\right) \mathrm{d} W_{2},
\end{aligned}
$$

where $W_{1,2}$ are cylindrical Wiener processes, the $\sigma, f, g, h$ are given functions, $B_{1,2}$ are operator-valued, $\Delta$ is the Laplace operator, $d>0$ is a parameter, the equation is posed on a bounded open domain $D \subset \mathbb{R}^{n}, u_{1,2}=u_{1,2}(x, t)$ are the unknowns for $(x, t) \in D \times\left[0, T_{\max }\right)$, and $T_{\max }$ is the maximal existence time. The term partly dissipative highlights the fact that only the first component contains the regularizing Laplace operator. In this work we analyse the case of additive noise and a certain coupling, more precisely,

$$
B_{1}\left(x, u_{1}, u_{2}\right)=B_{1}, \quad B_{2}\left(x, u_{1}, u_{2}\right)=B_{2}, \quad g\left(x, u_{1}, u_{2}\right)=g\left(x, u_{1}\right),
$$

We thank the anonymous referee for useful comments. CK and AN have been supported by a DFG grant in the D-A-CH framework (KU 3333/2-1). CK and AP acknowledge support by a Lichtenberg Professorship. 
where $B_{1,2}$ are bounded linear operators. A deterministic version of such a system has been analysed by Marion [20]. We are going to use certain assumptions for the reaction terms, which are similar to those used in [20]. The precise technical setting of our work starts in Sect. 2.

The goal of this work is to provide a general theory for stochastic partly dissipative systems and to analyse the long-time behaviour of the solution using the random dynamical systems approach. To this aim, we first show that the solution of our system exists globally-in-time, i.e. one can take $T_{\max }=+\infty$ above. Then we prove the existence of a pullback attractor. To our best knowledge the well-posedness and asymptotic behaviour for such systems (and for other coupled SPDEs and SODEs) has only been explored for special cases, i.e. mainly for the FitzHugh Nagumo equation, see $[4,24]$ for solution theory and $[2,19,31,32]$ for long-time behaviour/attractor theory. Here we develop a much more general theory of stochastic partly dissipative systems, motivated by the numerous applications in the natural sciences such as the the cubic-quintic Allen-Cahn equation [17] in elasticity. Moreover, unlike several previous works mentioned above, we deal with infinite-dimensional noise that satisfies certain regularity assumptions. These combined with the restrictions on the reaction terms allow us to compute sharp a-priori bounds of the solution, which are used to construct a random absorbing set. Even once the absorbing set has been constructed, we emphasize that we cannot directly apply compact embedding results to obtain the existence of an attractor. This issue arises due to the absence of the regularizing effect of the Laplacian in the second component. To overcome this obstacle, we introduce an appropriate splitting of the solution in two components: a regular one, and one that asymptotically tends to zero. This splitting technique goes (at least) back to Temam [28] and it has also been used in the context of deterministic partly dissipative systems [20] and for a stochastic FitzHugh-Nagumo equation with linear multiplicative noise [33,35]. The necessary additional technical steps for our setting are provided in Sect. 3.4. Using the a-priori bounds, we establish the existence of a pullback attractor $[9,14,25,26]$; which has been studied in several contexts to capture the long-time behaviour of stochastic (partial) differential equations, see for instance $[3,5,8,12,15]$ and the references therein. In the stochastic case pullback attractors are random invariant compact sets of phase space that are invariant with respect to the dynamics. They can be viewed as the generalization of non-autonomous attractors for deterministic systems. In the context of coupled SPDEs and SODEs, to our best knowledge, only random attractors for the stochastic FitzHugh-Nagumo equation were treated under various assumptions of the reaction and noise terms: finitedimensional additive noise on bounded and unbounded domains $[32,33]$ and for (non-autonomous) FitzHugh-Nagumo equation driven by linear multiplicative noise $[1,19,35]$. Here we provide a general random attractor theory for stochastic partly dissipative systems perturbed by infinite-dimensional additive noise, which goes beyond the FitzHugh-Nagumo system. To this aim we have to employ more general techniques than those used in the references specified above. Furthermore, we emphasize that other dynamical aspects for similar systems 
have been investigated, e.g. inertial manifolds and master-slave synchronization in reference [7].

We also mention that numerous extensions of our work are imaginable. Evidently the fully dissipative case is easier from the viewpoint of attractor theory. Hence, our results can be extended in a straightforward way to the case when both components of the SPDE contain a Laplacian. Systems with more than two components but with similar assumptions are likely just going to pose notational problems rather than intrinsic ones. From the point of view of applications it would be meaningful to incorporate non-linear couplings between the PDE and ODE parts. For example, this would allow us to use this theory to analyse various systems derived in chemical kinetics from massaction laws. However, more complicated non-linear couplings are likely to be far more challenging. Moreover, one could also develop a general framework which allows one to deal with other random influences, e.g. multiplicative noise, or more general Gaussian processes than standard trace-class Wiener processes. Furthermore, it would be interesting to investigate several dynamical aspects of partly dissipative SPDEs such as invariant manifolds or patterns. Naturally, one could also aim to derive upper bounds for the Hausdorff dimension of the random attractor and compare them to the deterministic result given in [20].

This paper is structured as follows: Sect. 2 contains all the preliminaries. More precisely, in Sect. 2.1 we define the system that we are going to analyse and state all the required assumptions. Subsequently, in Sect. 2.2, we clarify the notion of solution that we are interested in. The main contribution of this work is given in Sect. 3. Firstly, some preliminary definitions and results about random attractor theory are summarized in Sect. 3.1. Secondly, we derive the random dynamical system associated to our SPDE system in Sect. 3.2. Thirdly, we prove the existence of a bounded absorbing set for the random dynamical system in Sect. 3.3. Lastly, in Sect. 3.4 it is shown that one can indeed find a compact absorbing set implying the existence of a random attractor. In Sect. 4 we illustrate the theory by several examples arising from applications.

Notation. Before we start, we define/recall some standard notations that we will use within this work. When working with vectors we use $(\cdot)^{\top}$ to denote the transpose while $|\cdot|$ denotes the Euclidean norm. In a metric space $(M, d)$ we denote a ball of radius $r>0$ centred in the origin by

$$
B(r)=\{x \in M \mid d(x, 0) \leq r\} .
$$

We write Id for the identity operator/matrix. $L(U, H)$ denotes the space of bounded linear operators from $U$ to $H$. $O^{*}$ denotes the adjoint operator of a bounded linear operator $O$. We let $D \subset \mathbb{R}^{n}$ always be bounded, open, and with regular boundary, where $n \in \mathbb{N} . L^{p}(D), p \geq 1$, denotes the usual Lebesgue space with norm $\|\cdot\|_{p}$. Furthermore, $\langle\cdot, \cdot\rangle$ denotes the associated scalar-product in $L^{2}(D) . C^{p}(D), p \in \mathbb{N} \cup\{0, \infty\}$, denotes the space of all continuous functions that have continuous first $p$ derivatives. Lastly, for $k \in \mathbb{N}, 1 \leq p \leq \infty$ we consider the Sobolev space of order $k$ as

$$
W^{k, p}(D)=\left\{u \in L^{p}(D): D^{\alpha} u \in L^{p}(D) \forall|\alpha| \leqslant k\right\},
$$

with multi-index $\alpha$, where the norm is given by 


$$
\|u\|_{W^{k, p}(D)}:= \begin{cases}\left(\sum_{|\alpha| \leqslant k}\left\|D^{\alpha} u\right\|_{L^{p}(D)}^{p}\right)^{\frac{1}{p}} & 1 \leqslant p<\infty \\ \max _{|\alpha| \leqslant k}\left\|D^{\alpha} u\right\|_{L^{\infty}(D)} & p=\infty .\end{cases}
$$

The Sobolev space $W^{k, p}(D)$ is a Banach space. $H_{0}^{k}(D)$ denotes the space of functions in $H^{k}(D)=W^{k, 2}(D)$ that vanish at the boundary (in the sense of traces).

\section{Stochastic partly dissipative systems}

\subsection{Basics}

Let $D \subset \mathbb{R}^{n}$ be a bounded open set with regular boundary, set $H:=L^{2}(D)$ and let $U_{1}, U_{2}$ be two separable Hilbert spaces. We consider the following coupled, partly dissipative system with additive noise

$$
\begin{aligned}
& \mathrm{d} u_{1}=\left(d \Delta u_{1}-h\left(x, u_{1}\right)-f\left(x, u_{1}, u_{2}\right)\right) \mathrm{d} t+B_{1} \mathrm{~d} W_{1}, \\
& \mathrm{~d} u_{2}=\left(-\sigma(x) u_{2}-g\left(x, u_{1}\right)\right) \mathrm{d} t+B_{2} \mathrm{~d} W_{2},
\end{aligned}
$$

where $u_{1,2}=u_{1,2}(x, t),(x, t) \in D \times[0, T], T>0, W_{1,2}$ are cylindrical Wiener processes on $U_{1}$ respectively $U_{2}$, and $\Delta$ is the Laplace operator. Furthermore, $B_{1} \in L\left(U_{1}, H\right), B_{2} \in L\left(U_{2}, H\right)$ and $d>0$ is a parameter controlling the strength of the diffusion in the first component. The system is equipped with initial conditions

$$
u_{1}(x, 0)=u_{1}^{0}(x), u_{2}(x, 0)=u_{2}^{0}(x)
$$

and a Dirichlet boundary condition for the first component

$$
u_{1}(x, t)=0 \text { on } \partial D \times[0, T] .
$$

We will denote by $A$ the realization of the Laplace operator with Dirichlet boundary conditions, more precisely we define the operator $A: \mathcal{D}(A) \rightarrow L^{2}(D)$ as $A u=d \Delta u$ with domain $\mathcal{D}(A):=H^{2}(D) \cap H_{0}^{1}(D) \subset L^{2}(D)$. Note that $A$ is a self-adjoint operator that possesses a complete orthonormal system of eigenfunctions $\left\{e_{k}\right\}_{k=1}^{\infty}$ of $L^{2}(D)$. Within this work we always assume that there exists $\kappa>0$ such that $\left|e_{k}(x)\right|^{2}<\kappa$ for $k \in \mathbb{N}$ and $x \in D$. This holds for example when $D=[0, \pi]^{n}$. For the deterministic reaction terms appearing in (2.1)-(2.2) we assume that:

Assumption 2.1. (Reaction terms)

(1) $h \in C^{2}\left(\mathbb{R}^{n} \times \mathbb{R}\right)$ and there exist $\delta_{1}, \delta_{2}, \delta_{3}>0, p>2$ such that

$$
\delta_{1}\left|u_{1}\right|^{p}-\delta_{3} \leq h\left(x, u_{1}\right) u_{1} \leq \delta_{2}\left|u_{1}\right|^{p}+\delta_{3} .
$$

(2) $f \in C^{2}\left(\mathbb{R}^{n} \times \mathbb{R} \times \mathbb{R}\right)$ and there exist $\delta_{4}>0$ and $0<p_{1}<p-1$ such that

$$
\left|f\left(x, u_{1}, u_{2}\right)\right| \leq \delta_{4}\left(1+\left|u_{1}\right|^{p_{1}}+\left|u_{2}\right|\right) .
$$

(3) $\sigma \in C^{2}\left(\mathbb{R}^{n}\right)$ and there exist $\delta, \tilde{\delta}>0$ such that

$$
\delta \leq \sigma(x) \leq \tilde{\delta}
$$

(4) $g \in C^{2}\left(\mathbb{R}^{n} \times \mathbb{R}\right)$ and there exists $\delta_{5}>0$ such that

$$
\left|g_{u}\left(x, u_{1}\right)\right| \leq \delta_{5},\left|g_{x_{i}}\left(x, u_{1}\right)\right| \leq \delta_{5}\left(1+\left|u_{1}\right|\right), i=1, \ldots, n .
$$


In particular, Assumptions 2.1 (1) and (4) imply that there exist $\delta_{7}, \delta_{8}>$ 0 such that

$$
\begin{aligned}
& |g(x, \xi)| \leq \delta_{7}(1+|\xi|), \text { for all } \xi \in \mathbb{R}, x \in D, \\
& |h(x, \xi)| \leq \delta_{8}\left(1+|\xi|^{p-1}\right), \text { for all } \xi \in \mathbb{R}, x \in D .
\end{aligned}
$$

The Assumptions 2.1(1)-(4) are identical to those given in [20], except that in the deterministic case only a lower bound on $\sigma$ was assumed.

We always consider an underlying filtered probability space denoted as $\left(\Omega, \mathcal{F},\left(\mathcal{F}_{t}\right)_{t \geq 0}, \mathbb{P}\right)$ that will be specified later on. In order to guarantee certain regularity properties of the noise terms, we make the following additional assumptions:

\section{Assumption 2.2. (Noise)}

(1) We assume that $B_{2}: U_{2} \rightarrow H$ is a Hilbert-Schmidt operator. In particular, this implies that $Q_{2}:=B_{2} B_{2}^{*}$ is a trace class operator and $B_{2} W_{2}$ is a $Q_{2}$-Wiener process.

(2) We assume that $B_{1} \in L\left(U_{1}, H\right)$ and that the operator $Q_{t}$ defined by

$$
Q_{t} u=\int_{0}^{t} \exp (s A) Q_{1} \exp \left(s A^{*}\right) u \mathrm{~d} s, u \in H, t \geq 0,
$$

where $Q_{1}:=B_{1} B_{1}^{*}$, is of trace class. Hence, $B_{1} W_{1}$ is a $Q_{1}$-Wiener process as well.

(3) Let $U_{1}=H$. There exists an orthonormal basis $\left\{e_{k}\right\}_{k=1}^{\infty}$ of $H$ and sequences $\left\{\lambda_{k}\right\}_{k=1}^{\infty}$ and $\left\{\delta_{k}\right\}_{k=1}^{\infty}$ such that

$$
A e_{k}=-\lambda_{k} e_{k}, \quad Q_{1} e_{k}=\delta_{k} e_{k}, k \in \mathbb{N} .
$$

Furthermore, we assume that there exists $\alpha \in\left(0, \frac{1}{2}\right)$ such that

$$
\sum_{k=1}^{\infty} \delta_{k} \lambda_{k}^{2 \alpha+1}<\infty
$$

Assumptions 2.2 guarantee that the stochastic convolution introduced below is a well-defined process with sufficient regularity properties, see Lemmas 3.17 and 3.25. As an example, one could choose $B_{1}=(-A)^{-\gamma / 2}$ with $\gamma>\frac{n}{2}-1$ to ensure that Assumptions 2.2 (2)-(3) hold for $\alpha$ with $2 \alpha<\gamma-\frac{n}{2}+1$, see [10, Chapter 4].

Let us now formulate problem (2.1)-(2.2) as an abstract Cauchy problem. We define the following space

$$
\mathbb{H}:=L^{2}(D) \times L^{2}(D),
$$

with norm $\left\|\left(u_{1}, u_{2}\right)^{\top}\right\|_{\mathbb{H}}^{2}=\left\|u_{1}\right\|_{2}^{2}+\left\|u_{2}\right\|_{2}^{2}$ this becomes a separable Hilbert space. $\langle\cdot, \cdot\rangle_{\mathbb{H}}$ denotes the corresponding scalar product. Furthermore, we let

$$
\mathbb{V}:=H_{0}^{1}(D) \times L^{2}(D),
$$

with norm $\left\|\left(u_{1}, u_{2}\right)^{\top}\right\|_{\mathbb{V}}^{2}=\left\|u_{1}\right\|_{H^{1}(D)}^{2}+\left\|u_{2}\right\|_{2}^{2}$. We define the following linear operator

$$
\mathbf{A}:=\left(\begin{array}{cc}
A & 0 \\
0 & -\sigma(x)
\end{array}\right)
$$


where $\mathbf{A}: \mathcal{D}(\mathbf{A}) \subset \mathbb{H} \rightarrow \mathbb{H}$ with $\mathcal{D}(\mathbf{A})=\mathcal{D}(A) \times L^{2}(D)$. Since all the reaction terms are twice continuously differentiable they obey in particular the Carathéodory conditions [34]. Thus, the corresponding Nemytskii operator is defined by

$$
\begin{aligned}
\mathbf{F}\left(\left(u_{1}, u_{2}\right)^{\top}\right)(x) & :=\left(\begin{array}{c}
F_{1}\left(\left(u_{1}, u_{2}\right)^{\top}\right)(x) \\
F_{2}\left(\left(u_{1}, u_{2}\right)^{\top}\right)(x)
\end{array}\right), \\
& :=\left(\begin{array}{c}
-h\left(x, u_{1}(x)\right)-f\left(x, u_{1}(x), u_{2}(x)\right) \\
-g\left(x, u_{1}(x)\right)
\end{array}\right),
\end{aligned}
$$

where $\mathbf{F}: \mathcal{D}(\mathbf{F}) \subset \mathbb{H} \rightarrow \mathbb{H}$ and $\mathcal{D}(\mathbf{F}):=\mathbb{H}$. By setting

$$
\mathbf{W}:=\left(\begin{array}{l}
W_{1} \\
W_{2}
\end{array}\right), \quad \mathbf{B}:=\left(\begin{array}{l}
B_{1} \\
B_{2}
\end{array}\right), \quad \text { and } \quad u:=\left(\begin{array}{l}
u_{1} \\
u_{2}
\end{array}\right)
$$

we can rewrite the system (2.1)-(2.2) as an abstract Cauchy problem on the space $\mathbb{H}$

$$
\mathrm{d} u=(\mathbf{A} u+\mathbf{F}(u)) \mathrm{d} t+\mathbf{B} \mathrm{d} \mathbf{W},
$$

with initial condition

$$
u(0)=u^{0}:=\left(\begin{array}{l}
u_{1}^{0} \\
u_{2}^{0}
\end{array}\right)
$$

\subsection{Mild solutions and stochastic convolution}

We are interested in the concept of mild solutions to SPDEs. First of all, let us note the following. We have

$$
\mathbf{A}=\underbrace{\left(\begin{array}{ll}
A & 0 \\
0 & 0
\end{array}\right)}_{=: A_{1}}+\underbrace{\left(\begin{array}{cc}
0 & 0 \\
0 & -\sigma(x)
\end{array}\right)}_{=: A_{2}}
$$

It is well known that $A_{1}$ generates an analytic semigroup on $\mathbb{H}$ and $A_{2}$ is a bounded multiplication operator on $\mathbb{H}$. Hence, $\mathbf{A}$ is the generator of an analytic semigroup $\{\exp (t \mathbf{A})\}_{t \geq 0}$ on $\mathbb{H}$ as well, see [23, Chapter 3, Theorem 2.1]. Also note that $A$ generates an analytic semigroup $\{\exp (t A)\}_{t \geq 0}$ on $L^{p}(D)$ for every $p \geq 1$. In particular, we have for $u \in L^{p}(D)$ that for every $\alpha \geq 0$ there exists a constant $C_{\alpha}>0$ such that

$$
\left\|(-A)^{\alpha} \exp (t A) u\right\|_{p} \leq C_{\alpha} t^{-\alpha} \exp (a t)\|u\|_{p}, \text { for all } t>0,
$$

where $a>0$, see for instance [27, Theorem 37.5]. The domain $\mathcal{D}\left((-A)^{\alpha}\right)$ can be identified with the Sobolev space $W^{2 \alpha, p}(D)$ and thus we have in our setting for $t>0$

$$
\|\exp (t A) u\|_{W^{\alpha, p}(D)} \leq C_{\alpha} t^{-\alpha / 2} \exp (a t)\|u\|_{p} .
$$

Remark 2.3. Omitting the additive noise term in equation (2.11), we are in the deterministic setting of [20]. From there the existence of a global-in-time solution $\left(u_{1}, u_{2}\right) \in C([0, \infty), \mathbb{H})$ for every initial condition $u^{0} \in \mathbb{H}$ already follows. define

Let us now return to the stochastic Cauchy problem (2.11)-(2.12). We 
Definition 2.4. (Stochastic convolution) The stochastic process defined as

$$
W_{\mathbf{A}}(t):=\left(\begin{array}{l}
W_{\mathbf{A}}^{1}(t) \\
W_{\mathbf{A}}^{2}(t)
\end{array}\right):=\int_{0}^{t} \exp ((t-s) \mathbf{A}) \mathbf{B} \mathrm{d} \mathbf{W}(s),
$$

is called stochastic convolution.

More precisely, we have (see [22, Proposition 3.1])

$$
\begin{aligned}
W_{\mathbf{A}}(t) & =\int_{0}^{t}\left(\begin{array}{cc}
\exp ((t-s) A) & 0 \\
0 & \exp (-(t-s) \sigma(x))
\end{array}\right)\left(\begin{array}{l}
B_{1} \\
B_{2}
\end{array}\right) \mathrm{d} \mathbf{W}(s) \\
& =\left(\begin{array}{c}
\int_{0}^{t} \exp ((t-s) A) B_{1} \mathrm{~d} W_{1}(s) \\
\int_{0}^{t} \exp (-(t-s) \sigma(x)) B_{2} \mathrm{~d} W_{2}(s)
\end{array}\right) .
\end{aligned}
$$

This is a well-defined $\mathbb{H}$-valued Gaussian process. Furthermore, Assumptions 2.2 (1) and (2) ensure that $W_{\mathbf{A}}(t)$ is mean-square continuous and $\mathcal{F}_{t}$-measurable, see $[11]$.

Remark 2.5. As $W_{\mathbf{A}}$ is a Gaussian process, we can bound all its higher-order moments, i.e. for $p \geq 1$ we have

$$
\sup _{t \in[0, T]} \mathbb{E}\left\|W_{\mathbf{A}}(t)\right\|_{\mathbb{H}}^{p}<\infty .
$$

This follows from the Kahane-Khintchine inequality, see [29, Theorem 3.12].

Definition 2.6. (Mild solution) A mean-square continuous, $\mathcal{F}_{t}$-measurable $\mathbb{H}-$ valued process $u(t), t \in[0, T]$ is said to be a mild solution to $(2.11)-(2.12)$ on $[0, T]$ if $\mathbb{P}$-almost surely we have for $t \in[0, T]$

$$
u(t)=\exp (t \mathbf{A}) u^{0}+\int_{0}^{t} \exp ((t-s) \mathbf{A}) \mathbf{F}(u(s)) \mathrm{d} s+W_{\mathbf{A}}(t) .
$$

Under Assumptions 2.1 and $2.2(1)-(2)$ a mild solution exists locally-intime in

$$
L^{2}(\Omega ; C([0, T] ; \mathbb{H})) \cap L^{2}\left(\Omega ; L^{2}([0, T] ; \mathbb{V})\right),
$$

for some $T>0$, see [11, Theorem 7.7]. Hence, local in time existence for our problem is guaranteed by the classical SPDE theory.

\section{Random attractor}

\subsection{Preliminaries}

We now recall some basic definitions related to random attractors. For more information the reader is referred to the sources given in the introduction.

Definition 3.1. (Metric dynamical system) Let $(\Omega, \mathcal{F}, \mathbb{P})$ be a probability space and let $\theta=\left\{\theta_{t}: \Omega \rightarrow \Omega\right\}_{t \in \mathbb{R}}$ be a family of $\mathbb{P}$-preserving transformations (i.e. $\theta_{t} \mathbb{P}=\mathbb{P}$ for $\left.t \in \mathbb{R}\right)$, which satisfy for $t, s \in \mathbb{R}$ that

(1) $(t, \omega) \mapsto \theta_{t} \omega$ is measurable,

(2) $\theta_{0}=\mathrm{Id}$,

(3) $\theta_{t+s}=\theta_{t} \circ \theta_{s}$. 
Then $(\Omega, \mathcal{F}, \mathbb{P}, \theta)$ is called a metric dynamical system.

The metric dynamical system describes the dynamics of the noise.

Definition 3.2. (Random dynamical system) Let $(\mathcal{V},\|\cdot\|)$ be a separable Banach space. A random dynamical system $(\mathrm{RDS})$ with time domain $\mathbb{R}_{+}$on $(\mathcal{V},\|\cdot\|)$ over $\theta$ is a measurable map

$$
\varphi: \mathbb{R}_{+} \times \mathcal{V} \times \Omega \rightarrow \mathcal{V} ;(t, v, \omega) \mapsto \varphi(t, \omega) v
$$

such that $\varphi(0, \omega)=\operatorname{Id}_{\mathcal{V}}$ and

$$
\varphi(t+s, \omega)=\varphi\left(t, \theta_{s} \omega\right) \circ \varphi(s, \omega)
$$

for all $s, t \in \mathbb{R}_{+}$and for all $\omega \in \Omega$. We say that $\varphi$ is a continuous or differentiable $\operatorname{RDS}$ if $v \mapsto \varphi(t, \omega) v$ is continuous or differentiable for all $t \in \mathbb{R}_{+}$and every $\omega \in \Omega$.

We summarize some further definitions relevant for the theory of random attractors.

Definition 3.3. (Random set) A set-valued map $\mathcal{K}: \Omega \rightarrow 2^{\mathcal{V}}$ is said to be measurable if for all $v \in \mathcal{V}$ the map $\omega \mapsto d(v, \mathcal{K}(\omega))$ is measurable. Here, $d(\mathcal{A}, \mathcal{B})=\sup _{v \in \mathcal{A}} \inf _{\tilde{v} \in \mathcal{B}}\|v-\tilde{v}\|$ for $\mathcal{A}, \mathcal{B} \in 2^{\mathcal{V}}, \mathcal{A}, \mathcal{B} \neq \emptyset$ and $d(v, \mathcal{B})=$ $d(\{v\}, \mathcal{B})$. A measurable set-valued map is called a random set.

Definition 3.4. (Omega-limit set) For a random set $\mathcal{K}$ we define the omegalimit set to be

$$
\Omega_{\mathcal{K}}(\omega):=\bigcap_{T \geq 0} \overline{\bigcup_{t \geq T} \varphi\left(t, \theta_{-t} \omega\right) \mathcal{K}\left(\theta_{-t} \omega\right)} .
$$

$\Omega_{\mathcal{K}}(\omega)$ is closed by definition.

Definition 3.5. (Attracting and absorbing set) Let $\mathcal{A}, \mathcal{B}$ be random sets and let $\varphi$ be a RDS.

- $\mathcal{B}$ is said to attract $\mathcal{A}$ for the $\operatorname{RDS} \varphi$, if

$$
d\left(\varphi\left(t, \theta_{-t} \omega\right) \mathcal{A}\left(\theta_{-t} \omega\right), \mathcal{B}(\omega)\right) \rightarrow 0 \text { for } t \rightarrow \infty .
$$

- $\mathcal{B}$ is said to absorb $\mathcal{A}$ for the $\operatorname{RDS} \varphi$, if there exists a (random) absorption time $t_{\mathcal{A}}(\omega)$ such that for all $t \geq t_{\mathcal{A}}(\omega)$

$$
\varphi\left(t, \theta_{-t} \omega\right) \mathcal{A}\left(\theta_{-t} \omega\right) \subset \mathcal{B}(\omega) .
$$

- Let $\mathcal{D}$ be a collection of random sets (of non-empty subsets of $\mathcal{V}$ ), which is closed with respect to set inclusion. A set $\mathcal{B} \in \mathcal{D}$ is called $\mathcal{D}$-absorbing $/ \mathcal{D}$ attracting for the $\operatorname{RDS} \varphi$, if $\mathcal{B}$ absorbs/attracts all random sets in $\mathcal{D}$.

Remark 3.6. Throughout this work we use a convenient criterion to derive the existence of an absorbing set. Let $\mathcal{A}$ be a random set. If for every $v \in \mathcal{A}\left(\theta_{-t} \omega\right)$ we have

$$
\limsup _{t \rightarrow \infty}\left\|\varphi\left(t, \theta_{-t} \omega, v\right)\right\| \leq \rho(\omega),
$$

where $\rho(\omega)>0$ for every $\omega \in \Omega$, then the ball centred in 0 with radius $\rho(\omega)+\epsilon$ for a $\epsilon>0$, i.e. $\mathcal{B}(\omega):=B(0, \rho(\omega)+\epsilon)$, absorbs $\mathcal{A}$. 
Definition 3.7. (Tempered set) A random set $\mathcal{A}$ is called tempered provided for $\mathbb{P}$-a.e. $\omega \in \Omega$

$$
\lim _{t \rightarrow \infty} \exp (-\beta t) d\left(\mathcal{A}\left(\theta_{-t} \omega\right)\right)=0 \text { for all } \beta>0,
$$

where $d(\mathcal{A})=\sup _{a \in \mathcal{A}}\|a\|$. We denote by $\mathcal{T}$ the set of all tempered subsets of $\mathcal{V}$.

Definition 3.8. (Tempered random variable) A random variable $X \in \mathbb{R}$ on $(\Omega, \mathcal{F}, \mathbb{P}, \theta)$ is called tempered, if there is a set of full $\mathbb{P}$-measure such that for all $\omega$ in this set we have

$$
\lim _{t \rightarrow \pm \infty} \frac{\log \left|X\left(\theta_{t} \omega\right)\right|}{|t|}=0 .
$$

Hence a random variable $X$ is tempered when the stationary random process $X\left(\theta_{t} \omega\right)$ grows sub-exponentially.

Remark 3.9. A sufficient condition that a positive random variable $X$ is tempered is that (cf. [3, Proposition 4.1.3])

$$
\mathbb{E}\left(\sup _{t \in[0,1]} X\left(\theta_{t} \omega\right)\right)<\infty .
$$

If $\theta$ is an ergodic shift, then the only alternative to $(3.2)$ is

$$
\lim _{t \rightarrow \pm \infty} \frac{\log \left|X\left(\theta_{t} \omega\right)\right|}{|t|}=\infty,
$$

i.e., the random process $X\left(\theta_{t} \omega\right)$ either grows sub-exponentially or blows up at least exponentially.

Definition 3.10. (Random attractor) Suppose $\varphi$ is a RDS such that there exists a random compact set $\mathcal{A} \in \mathcal{T}$ which satisfies for any $\omega \in \Omega$

- $\mathcal{A}$ is invariant, i.e., $\varphi(t, \omega) \mathcal{A}(\omega)=\mathcal{A}\left(\theta_{t} \omega\right)$ for all $t \geq 0$.

- $\mathcal{A}$ is $\mathcal{T}$-attracting.

Then $\mathcal{A}$ is said to be a $\mathcal{T}$-random attractor for the RDS.

Theorem 3.11. $([9,25])$ Let $\varphi$ be a continuous $R D S$ and assume there exists a compact random set $\mathcal{B} \in \mathcal{T}$ that absorbs every $\mathcal{D} \in \mathcal{T}$, i.e. $\mathcal{B}$ is $\mathcal{T}$-absorbing. Then there exists a unique $\mathcal{T}$-random attractor $\mathcal{A}$, which is given by

$$
\mathcal{A}(\omega)=\overline{\bigcup_{\mathcal{D} \in \mathcal{T}} \Omega_{\mathcal{D}}(\omega)} .
$$

We will use the above theorem to show the existence of a random attractor for the partly dissipative system at hand. 


\subsection{Associated RDS}

We will now define the RDS corresponding to (2.11)-(2.12). We consider $\mathcal{V}=$ $\mathbb{H}:=L^{2}(D) \times L^{2}(D)$ and $\mathcal{T}$ is the set of all tempered subsets of $\mathbb{H}$. In the sequel, we consider the fixed canonical probability space $(\Omega, \mathcal{F}, \mathbb{P})$ corresponding to a two-sided Wiener process, more precisely

$$
\Omega:=\left\{\omega=\left(\omega_{1}, \omega_{2}\right): \omega_{1}, \omega_{2} \in C\left(\mathbb{R}, L^{2}(D)\right), \omega(0)=0\right\},
$$

endowed with the compact-open topology. The $\sigma$-algebra $\mathcal{F}$ is the Borel $\sigma$ algebra on $\Omega$ and $\mathbb{P}$ is the distribution of the trace class Wiener process $\tilde{W}(t):=$ $\left(\tilde{W}_{1}(t), \tilde{W}_{2}(t)\right)=\left(B_{1} W_{1}(t), B_{2} W_{2}(t)\right)$, where we recall that $B_{1}$ and $B_{2}$ fulfil Assumptions 2.2. We identify the elements of $\Omega$ with the paths of these Wiener processes, more precisely

$$
\tilde{W}(t, \omega):=\left(\tilde{W}_{1}\left(t, \omega_{1}\right), \tilde{W}_{2}\left(t, \omega_{2}\right)\right)=\left(\omega_{1}(t), \omega_{2}(t)\right)=: \omega(t), \text { for } \omega \in \Omega .
$$

Furthermore, we introduce the Wiener shift, namely

$$
\theta_{t} \omega(\cdot):=\omega(\cdot+t)-\omega(t), \text { for } \omega \in \Omega \text { and } t \in \mathbb{R} .
$$

Then $\theta: \mathbb{R} \times \Omega \rightarrow \Omega$ is a measure-preserving transformation on $\Omega$, i.e. $\theta_{t} \mathbb{P}=\mathbb{P}$, for $t \in \mathbb{R}$. Furthermore, $\theta_{0} \omega(s)=\omega(s)-\omega(0)=\omega(s)$ and $\theta_{t+s} \omega(r)=\omega(r+$ $t+s)-\omega(t+s)=\theta_{t}(\omega(r+s)-\omega(s))=\theta_{t}\left(\theta_{s} \omega(r)\right)$. Hence, $(\Omega, \mathcal{F}, \mathbb{P}, \theta)$ is a metric dynamical system. Next, we consider the following equations

$$
\begin{aligned}
& \mathrm{d} z_{1}=A z_{1} \mathrm{~d} t+\mathrm{d} \omega_{1}, \\
& \mathrm{~d} z_{2}=-\sigma(x) z_{2} \mathrm{~d} t+\mathrm{d} \omega_{2} .
\end{aligned}
$$

The stationary solutions of (3.6)-(3.7) are given by

$$
(t, \omega) \mapsto z_{1}\left(\theta_{t} \omega\right) \text { and }(t, \omega) \mapsto z_{2}\left(\theta_{t} \omega\right),
$$

where

$$
\begin{aligned}
& z_{1}\left(\theta_{t} \omega\right)=\int_{-\infty}^{t} e^{(t-s) A} \mathrm{~d} \omega_{1}(s)=\int_{-\infty}^{0} e^{-s A} \mathrm{~d} \theta_{t} \omega_{1}(s), \\
& z_{2}\left(\theta_{t} \omega\right)=\int_{-\infty}^{t} e^{-(t-s) \sigma(x)} \mathrm{d} \omega_{2}(s)=\int_{-\infty}^{0} e^{s \sigma(x)} \mathrm{d} \theta_{t} \omega_{2}(s) .
\end{aligned}
$$

Here, we observe that for $t=0$

$$
z_{1}(\omega)=\int_{-\infty}^{0} e^{-s A} \mathrm{~d} \omega_{1}(s), z_{2}(\omega)=\int_{-\infty}^{0} e^{s \sigma(x)} \mathrm{d} \omega_{2}(s) .
$$

Now consider the Doss-Sussmann transformation $v(t)=u(t)-z\left(\theta_{t} \omega\right)$, where $v(t)=\left(v_{1}(t), v_{2}(t)\right)^{\top}, z(\omega)=\left(z_{1}\left(\omega_{1}\right), z_{2}\left(\omega_{2}\right)\right)^{\top}$ and $u(t)=\left(u_{1}(t), u_{2}(t)\right)^{\top}$ is a solution to the problem (2.1)-(2.4). Then $v(t)$ satisfies

$$
\frac{\mathrm{d} v}{\mathrm{~d} t}=\mathbf{A} v+\mathbf{F}\left(v+z\left(\theta_{t} \omega\right)\right) .
$$


More explicitly/or component-wise this reads as

$$
\begin{aligned}
\frac{\mathrm{d} v_{1}(t)}{\mathrm{d} t}= & d \Delta v_{1}(t)-h\left(x, v_{1}(t)+z_{1}\left(\theta_{t} \omega\right)\right) \\
& -f\left(x, v_{1}(t)+z_{1}\left(\theta_{t} \omega\right), v_{2}(t)+z_{2}\left(\theta_{t} \omega\right)\right), \\
\frac{\mathrm{d} v_{2}(t)}{\mathrm{d} t}= & -\sigma(x) v_{2}(t)-g\left(x, v_{1}(t)+z_{1}\left(\theta_{t} \omega\right)\right) .
\end{aligned}
$$

In the equations above no stochastic differentials appear, hence they can be considered path-wise, i.e., for every $\omega$ instead just for $\mathbb{P}$-almost every $\omega$. For every $\omega(3.8)$ is a deterministic equation, where $z\left(\theta_{t} \omega\right)$ can be regarded as a time-continuous perturbation. In particular, [6] guarantees that for all $v^{0}=\left(v_{1}^{0}, v_{2}^{0}\right)^{\top} \in \mathbb{H}$ there exists a solution $v\left(\cdot, \omega, v^{0}\right) \in C([0, \infty), \mathbb{H})$ with $v_{1}\left(0, \omega, v_{1}^{0}\right)=v_{1}^{0}, v_{2}\left(0, \omega, v_{2}^{0}\right)=v_{2}^{0}$. Moreover, the mapping $\mathbb{H} \ni v_{0} \mapsto v\left(t, \omega, v_{0}\right) \in$ $\mathbb{H}$ is continuous. Now, let

$$
\begin{aligned}
& u_{1}\left(t, \omega, u_{1}^{0}\right)=v_{1}\left(t, \omega, u_{1}^{0}-z_{1}(\omega)\right)+z_{1}\left(\theta_{t} \omega\right), \\
& u_{2}\left(t, \omega, u_{2}^{0}\right)=v_{2}\left(t, \omega, u_{2}^{0}-z_{2}(\omega)\right)+z_{2}\left(\theta_{t} \omega\right) .
\end{aligned}
$$

Then $u\left(t, \omega, u^{0}\right)=\left(u_{1}\left(t, \omega, u_{1}^{0}\right), u_{2}\left(t, \omega, u_{2}^{0}\right)\right)^{\top}$ is a solution to $(2.1)-(2.4)$. In particular, we can conclude at this point that (2.1)-(2.4) has a global-in-time solution which belongs to $C([0, \infty) ; \mathbb{H})$; see Remark 2.3. We define the corresponding solution operator $\varphi: \mathbb{R}^{+} \times \Omega \times \mathbb{H} \rightarrow \mathbb{H}$ as

$$
\varphi\left(t, \omega,\left(u_{1}^{0}, u_{2}^{0}\right)\right):=\left(u_{1}\left(t, \omega, u_{1}^{0}\right), u_{2}\left(t, \omega, u_{2}^{0}\right)\right),
$$

for all $\left(t, \omega,\left(u_{1}^{0}, u_{2}^{0}\right)\right) \in \mathbb{R}^{+} \times \Omega \times \mathbb{H}$. Now, $\varphi$ is a continuous RDS associated to our stochastic partly dissipative system. In particular, the cocycle property obviously follows from the mild formulation. In the following, we will prove the existence of a global random attractor for this RDS. Due to conjugacy, see $[9,25]$ this gives us automatically a global random attractor for the stochastic partly dissipative system (2.1)-(2.4).

\subsection{Bounded absorbing set}

In the following we will prove the existence of a bounded absorbing set for the RDS (3.11). In the calculations we will make use of some versions of certain classical deterministic results several times. Therefore, we recall these results here for completeness and as an aid to follow the calculations later on.

Lemma 3.12. ( $\varepsilon$-Young inequality) For $x, y \in \mathbb{R}, \varepsilon>0, \tilde{p}, \tilde{q}>1, \frac{1}{\tilde{p}}+\frac{1}{\tilde{q}}=1$ we have

$$
|x y| \leq \varepsilon|x|^{\tilde{p}}+\frac{(\tilde{p} \varepsilon)^{1-\tilde{q}}}{\tilde{q}}|y|^{\tilde{q}} .
$$

Lemma 3.13. (Gronwall's inequality) Assume that $\varphi, \alpha$ and $\beta$ are integrable functions and $\varphi(t) \geq 0$. If

$$
\varphi^{\prime}(t) \leq \alpha(t)+\beta(t) \varphi(t),
$$

then

$$
\varphi(t) \leq \varphi\left(t_{0}\right) \exp \left(\int_{t_{0}}^{t} \beta(\tau) d \tau\right)+\int_{t_{0}}^{t} \alpha(s) \exp \left(\int_{s}^{t} \beta(\tau) d \tau\right) d s, t \geq t_{0} .
$$


Lemma 3.14. (Uniform Gronwall Lemma [28, Lemma 1.1]) Let $g$, h, y be positive locally integrable functions on $\left(t_{0}, \infty\right)$ such that $y^{\prime}$ is locally integrable on $\left(t_{0}, \infty\right)$ and which satisfy

$$
\begin{aligned}
& \frac{\mathrm{d} y}{\mathrm{~d} t} \leq g y+h, \quad \text { for } t \geq t_{0}, \\
& \int_{t}^{t+r} g(s) \mathrm{d} s \leq a_{1}, \quad \int_{t}^{t+r} h(s) \mathrm{d} s \leq a_{2}, \quad \int_{t}^{t+r} y(s) \mathrm{d} s \leq a_{3} \quad \text { for } t \geq t_{0},
\end{aligned}
$$

where $r, a_{1}, a_{2}, a_{3}$ are positive constants. Then

$$
y(t+r) \leq\left(\frac{a_{3}}{r}+a_{2}\right) \exp \left(a_{1}\right), \forall t \geq t_{0}
$$

Lemma 3.15. (Minkowski's inequality) Let $p>1$ and $f, g \in \mathbb{R}$, then

$$
|f+g|^{p} \leq 2^{p-1}\left(|f|^{p}+|g|^{p}\right)
$$

Lemma 3.16. (Poincaré's inequality) Let $1 \leq p<\infty$ and let $D \subset \mathbb{R}^{n}$ be a bounded open subset. Then there exists a constant $c=c(D, p)$ such that for every function $u \in W_{0}^{1, p}(D)$

$$
\|u\|_{p} \leq c\|\nabla u\|_{p}
$$

Having recalled the relevant deterministic preliminaries, we can now proceed with the main line of our argument. For the following result about the stochastic convolutions Assumption 2.2 (3) is crucial.

Lemma 3.17. Suppose Assumptions 2.1 and 2.2 hold. Then for every $p \geq 1$

$$
\left\|z_{1}(\omega)\right\|_{p}^{p} \text { and }\left\|z_{2}(\omega)\right\|_{2}^{2}
$$

are tempered random variables. 
Proof. Using $0<\delta \leq \sigma(x) \leq \tilde{\delta}$ and the Burkholder-Davis-Gundy inequality we have

$$
\begin{aligned}
& \mathbb{E}\left(\sup _{t \in[0,1]}\left\|z_{2}\left(\theta_{t} \omega\right)\right\|_{2}^{2}\right) \\
& \quad=\mathbb{E}\left(\sup _{t \in[0,1]}\left\|\int_{-\infty}^{t} \exp (-(t-s) \sigma(x)) \mathrm{d} \omega_{2}(s)\right\|_{2}^{2}\right) \\
& \quad=\mathbb{E}\left(\sup _{t \in[0,1]} \int_{D} \exp (-2 t \sigma(x))\left|\int_{-\infty}^{t} \exp (s \sigma(x)) \mathrm{d} \omega_{2}(s)\right|^{2} \mathrm{~d} x\right) \\
& \leq \mathbb{E}\left(\sup _{t \in[0,1]} \exp (-2 t \delta) \int_{D}\left|\int_{-\infty}^{t} \exp (s \sigma(x)) \mathrm{d} \omega_{2}(s)\right|^{2} \mathrm{~d} x\right) \\
& \leq \mathbb{E}\left(\sup _{t \in[0,1]}\left\|\int_{-\infty}^{t} \exp (s \sigma(x)) \mathrm{d} \omega_{2}(s)\right\|_{2}^{2}\right) \\
& \quad \leq C \mathbb{E}\left(\int_{-\infty}^{1}\|\exp (s \sigma(x))\|_{2}^{2} \mathrm{~d} s\right) \\
& \quad \leq C|D| \int_{-\infty}^{1} \exp (2 s \tilde{\delta}) \mathrm{d} s=\frac{C|D|}{2 \tilde{\delta}} \exp (2 \tilde{\delta}) \\
& \quad<\infty
\end{aligned}
$$

The temperedness of $\left\|z_{2}(\omega)\right\|_{2}^{2}$ then follows directly using Remark 3.9. Now, we consider the random variable $\left\|z_{1}(\omega)\right\|_{p}^{p}$. Note that using the so-called factorization method we have for $(x, t) \in D \times[0, T]$ and $\alpha \in(0,1 / 2)$ (see [11, Ch. $5.3])$

$$
z_{1}\left(x, \theta_{t} \omega\right)=\frac{\sin (\pi \alpha)}{\pi} \int_{-\infty}^{t} \exp ((t-\tau) A)(t-\tau)^{\alpha-1} Y(x, \tau) \mathrm{d} \tau,
$$

with

$$
\begin{aligned}
Y(x, \tau) & =\int_{0}^{\tau} \exp ((\tau-s) A)(\tau-s)^{-\alpha} B_{1} \mathrm{~d} W_{1}(x, s) \\
& =\sum_{k=1}^{\infty} \int_{0}^{\tau} \exp ((\tau-s) A)(\tau-s)^{-\alpha} B_{1} e_{k}(x) d \beta_{k}(s) \\
& =\sum_{k=1}^{\infty} \int_{0}^{\tau} \exp \left(-(\tau-s) \lambda_{k}\right)(\tau-s)^{-\alpha} \sqrt{\delta_{k}} e_{k}(x) d \beta_{k}(s),
\end{aligned}
$$

where we have used the formal representation $W_{1}(x, s)=\sum_{k=1}^{\infty} \beta_{k}(s) e_{k}(x)$ of the cylindrical Wiener process, with $\left\{\beta_{k}\right\}_{k=1}^{\infty}$ being a sequence of mutually independent real-valued Brownian motions. $Y(x, \tau)$ is a real-valued Gaussian random variable with mean zero and variance 


$$
\begin{aligned}
\operatorname{Var} & (Y(x, \tau))=\mathbb{E}\left[|Y(x, \tau)|^{2}\right] \\
& =\mathbb{E}\left[\sum_{k=1}^{\infty}\left(\int_{0}^{\tau} \exp \left(-(\tau-s) \lambda_{k}\right)(t-s)^{-\alpha} \sqrt{\delta_{k}} \mathrm{~d} \beta_{k}(s)\right)^{2}\left|e_{k}(x)\right|^{2}\right] \\
& =\sum_{k=1}^{\infty} \delta_{k}\left|e_{k}(x)\right|^{2} \mathbb{E}\left[\left(\int_{0}^{\tau} \exp \left(-(\tau-s) \lambda_{k}\right)(t-s)^{-\alpha} \mathrm{d} \beta_{k}(s)\right)^{2}\right] \\
& =\sum_{k=1}^{\infty} \delta_{k}\left|e_{k}(x)\right|^{2} \int_{0}^{\tau} \exp \left(-2 s \lambda_{k}\right) s^{-2 \alpha} \mathrm{d} s,
\end{aligned}
$$

where we have used Parseval's identity and the Itô isometry. Our assumption on the boundedness of the eigenfunctions $\left\{e_{k}\right\}_{k=1}^{\infty}$ yields together with Assumption 2.2 (3) that

$$
\begin{aligned}
\operatorname{Var}(Y(x, \tau)) & <\sum_{k=1}^{\infty} \delta_{k} \kappa^{2} \int_{0}^{\infty} \exp \left(-2 s \lambda_{k}\right) s^{-2 \alpha} \mathrm{d} s \\
& =\kappa^{2} 2^{2 \alpha-1} \Gamma(1-2 \alpha) \sum_{k=1}^{\infty} \delta_{k} \lambda_{k}^{2 \alpha-1}<\infty
\end{aligned}
$$

Hence, $\mathbb{E}\left[|Y(x, \tau)|^{2 m}\right] \leq C_{m}$ for $C_{m}>0$ and every $m \geq 1$ (note that all odd moments of a Gaussian random variable are zero). Thus we have

$$
\mathbb{E}\left[\int_{0}^{T} \int_{D}|Y(x, \tau)|^{2 m} \mathrm{~d} x \mathrm{~d} \tau\right] \leq T C_{m}|D|,
$$

i.e., in particular for all $p \geq 1$ we have $Y \in L^{p}(D \times[0, T]) \mathbb{P}$-a.s.. We now observe

$$
\begin{aligned}
& \left\|z_{1}\left(\theta_{t} \omega\right)\right\|_{W^{\alpha, p}(D)} \\
& \quad \leq \frac{\sin (\pi \alpha)}{\pi} \int_{-\infty}^{t}(t-\tau)^{\alpha-1}\|\exp ((t-\tau) A) Y(\cdot, \tau)\|_{W^{\alpha, p}(D)} \mathrm{d} \tau \\
& \quad \leq C \frac{\sin (\pi \alpha)}{\pi} \int_{-\infty}^{t}(t-\tau)^{\alpha-1}(t-\tau)^{-\alpha / 2} e^{-\lambda(t-\tau)}\|Y(\cdot, \tau)\|_{p} \mathrm{~d} \tau \\
& \quad \leq C \sup _{\tau \in(-\infty, t]}\|Y(\cdot, \tau)\|_{p} \int_{-\infty}^{t}(t-\tau)^{\alpha / 2-1} e^{-\lambda(t-\tau)} \mathrm{d} \tau,
\end{aligned}
$$

where we have used (2.13) and thus

$$
\begin{aligned}
& \mathbb{E}\left(\sup _{t \in[0,1]}\left\|z_{1}\left(\theta_{t} \omega\right)\right\|_{p}\right) \\
& \leq C \mathbb{E}\left(\sup _{t \in[0,1]} \sup _{\tau \in(-\infty, t]}\|Y(\cdot, \tau)\|_{p}\right) \int_{0}^{\infty} \tau^{\alpha / 2-1} e^{-\lambda \tau} \mathrm{d} \tau \\
& =C \mathbb{E}\left(\sup _{t \in[0,1]} \sup _{\tau \in(-\infty, t]}\|Y(\cdot, \tau)\|_{p}\right) \frac{\Gamma(\alpha / 2)}{\lambda^{\alpha / 2}} .
\end{aligned}
$$


Now, the right hand side is finite as all moments of $Y(x, \tau)$ are bounded uniformly in $x, \tau$, see above. Due to embedding of Lebesgue spaces on a bounded domain we have that

$$
\mathbb{E}\left(\sup _{t \in[0,1]}\left\|z_{1}\left(\theta_{t} \omega\right)\right\|_{p}\right)<\infty \text { implies } \mathbb{E}\left(\sup _{t \in[0,1]}\left\|z_{1}\left(\theta_{t} \omega\right)\right\|_{p}^{p}\right)<\infty,
$$

i.e., temperedness of $\left\|z_{1}(\omega)\right\|_{p}^{p}$ follows again with Remark 3.9.

Remark 3.18. (1) Note that Assumption 2.2 (3) together with the boundedness of $e_{k}$ for $k \in \mathbb{N}$ are essential for this proof. One can extend such statements for general open bounded domains in $D \subset \mathbb{R}^{n}$, according to Remark 5.27 and Theorem 5.28 in [11].

(2) Regarding again Assumption 2.2 (3) one can show in a similar way that $z_{1} \in W^{1, p}(D)$ and in particular also $\left\|\nabla z_{1}(\omega)\right\|_{p}^{p}$ is a tempered random variable for all $p \geq 1$.

Remark 3.19. Alternatively, one can introduce the Ornstein-Uhlenbeck processes $z_{1}$ and $z_{2}$ using integration by parts. We applied the factorization Lemma for the definition of $z_{1}$ in order to obtain regularity results for $z_{1}$ based on the interplay between the eigenvalues of the linear part and of the covariance operator of the noise.

Using integration by parts, one infers that

$$
\begin{aligned}
z_{1}\left(\theta_{t} \omega\right) & =\int_{-\infty}^{t} \exp ((t-\tau) A) \mathrm{d} \omega_{1}(\tau)=\omega_{1}(t)+A \int_{-\infty}^{t} \exp ((t-\tau) A) \omega_{1}(\tau) \mathrm{d} \tau \\
& =-A \int_{-\infty}^{t} \exp ((t-\tau) A)\left(\omega_{1}(t)-\omega_{1}(\tau)\right) \mathrm{d} \tau
\end{aligned}
$$

This expression can also be used in order to investigate the regularity of $z_{1}$ in a Banach space $\mathcal{H}$ as follows:

$$
\begin{aligned}
& \left\|A \int_{-\infty}^{t} \exp ((t-\tau) A)\left(\omega_{1}(t)-\omega_{1}(\tau)\right) \mathrm{d} \tau\right\|_{\mathcal{H}} \\
& \quad \leq C \int_{-\infty}^{t}(t-\tau)^{-1}\|\exp (t-\tau) A\|_{\mathcal{H}}\left\|\omega_{1}(t)-\omega_{1}(\tau)\right\|_{\mathcal{H}} \mathrm{d} \tau .
\end{aligned}
$$

Here one uses the Hölder-continuity of $\omega_{1}$ in an appropriate function space in order to compensate the singularity in the previous formula.

In our case, we need $z_{1} \in D\left(A^{\alpha / 2}\right)=W^{\alpha, p}(D)$. Letting $\omega_{1} \in D\left(A^{\varepsilon}\right)$ for $\varepsilon \geq 0$ and using that $\omega_{1}$ is $\beta$-Hölder continuous with $\beta \leq 1 / 2$ one has

$$
\left\|z_{1}\left(\theta_{t} \omega\right)\right\|_{W^{\alpha, p}(D)} \leq \int_{-\infty}^{t}(t-\tau)^{\beta+\varepsilon-\alpha / 2-1}\left\|\omega_{1}\right\|_{\beta, \varepsilon}\|\exp ((t-\tau) A)\| \mathrm{d} \tau,
$$

which is well-defined if $\beta+\varepsilon>\alpha / 2$. Such a condition provides again an interplay between the time and space regularity of the stochastic convolution.

Based on the results regarding the stochastic convolutions we can now investigate the long-time behaviour of our system. The first step is contained in the next lemma, which establishes the existence of an absorbing set. 
Lemma 3.20. Suppose Assumptions 2.1 and 2.2 hold. Then there exists a set $\{\mathcal{B}(\omega)\}_{\omega \in \Omega} \in \mathcal{T}$ such that $\{\mathcal{B}(\omega)\}_{\omega \in \Omega}$ is a bounded absorbing set for $\varphi$. In particular, for any $\mathcal{D}=\{\mathcal{D}(\omega)\}_{\omega \in \Omega} \in \mathcal{T}$ and every $\omega \in \Omega$ there exists a random time $t_{\mathcal{D}}(\omega)$ such that for all $t \geq t_{\mathcal{D}}(\omega)$

$$
\varphi\left(t, \theta_{-t} \omega, \mathcal{D}\left(\theta_{-t} \omega\right)\right) \subset \mathcal{B}(\omega)
$$

Proof. To show the existence of a bounded absorbing set, we want to make use of Remark 3.6, i.e. we need an a-priori estimate in $\mathbb{H}$. We have for $v=\left(v_{1}, v_{2}\right)^{\top}$ solution of (3.8)

$$
\begin{aligned}
\frac{1}{2} \frac{\mathrm{d}}{\mathrm{d} t} & \left(\left\|v_{1}\right\|_{2}^{2}+\left\|v_{2}\right\|_{2}^{2}\right)=\frac{1}{2} \frac{\mathrm{d}}{\mathrm{d} t}\|v\|_{\mathbb{H}}^{2}=\left\langle\frac{\mathrm{d}}{\mathrm{d} t} v, v\right\rangle_{\mathbb{H}}=\left\langle\mathbf{A} v+\mathbf{F}\left(v+z\left(\theta_{t} \omega\right)\right), v\right\rangle_{\mathbb{H}} \\
= & \left\langle d A v_{1}, v_{1}\right\rangle+\left\langle F_{1}\left(v+z\left(\theta_{t} \omega\right)\right), v_{1}\right\rangle-\left\langle\sigma(x) v_{2}, v_{2}\right\rangle+\left\langle F_{2}\left(v+z\left(\theta_{t} \omega\right)\right), v_{2}\right\rangle \\
= & -d\left\|\nabla v_{1}\right\|_{2}^{2} \underbrace{-\left\langle h\left(x, v_{1}+z_{1}\left(\theta_{t} \omega\right)\right), v_{1}\right\rangle}_{=: I_{1}} \underbrace{-\left\langle f\left(x, v_{1}+z_{1}\left(\theta_{t} \omega\right), v_{2}+z_{2}\left(\theta_{t} \omega\right)\right), v_{1}\right\rangle}_{=: I_{2}} \\
& -\delta\left\|v_{2}\right\|_{2}^{2} \underbrace{-\left\langle g\left(x, v_{1}+z_{1}\left(\theta_{t} \omega\right)\right), v_{2}\right\rangle}_{=: I_{3}},
\end{aligned}
$$

where we have used (2.7). We now estimate $I_{1}-I_{3}$ separately. Deterministic constants denoted as $C, C_{1}, C_{2}, \ldots$ may change from line to line. Using (2.5) and (2.10) we calculate

$$
\begin{aligned}
I_{1}= & -\int_{D} h\left(x, v_{1}+z_{1}\left(\theta_{t} \omega\right)\right) v_{1} \mathrm{~d} x \\
= & -\int_{D} h\left(x, v_{1}+z_{1}\left(\theta_{t} \omega\right)\right)\left(v_{1}+z_{1}\left(\theta_{t} \omega\right)\right) \mathrm{d} x \\
& +\int_{D} h\left(x, v_{1}+z_{1}\left(\theta_{t} \omega\right)\right) z_{1}\left(\theta_{t} \omega\right) \mathrm{d} x \\
\leq & -\int_{D} \delta_{1}\left|u_{1}\right|^{p} \mathrm{~d} x+\int_{D} \delta_{3} \mathrm{~d} x+\int_{D}\left|h\left(x, v_{1}+z_{1}\left(\theta_{t} \omega\right)\right) \| z_{1}\left(\theta_{t} \omega\right)\right| \mathrm{d} x \\
\leq & -\delta_{1}\left\|u_{1}\right\|_{p}^{p}+C+\delta_{8} \int_{D}\left(1+\left|u_{1}\right|^{p-1}\right)\left|z_{1}\left(\theta_{t} \omega\right)\right| \mathrm{d} x \\
= & -\delta_{1}\left\|u_{1}\right\|_{p}^{p}+C+\delta_{8}\left\|z_{1}\left(\theta_{t} \omega\right)\right\|_{1}+\delta_{8} \int\left|u_{1}\right|^{p-1}\left|z_{1}\left(\theta_{t} \omega\right)\right| \mathrm{d} x \\
\leq & -\delta_{1}\left\|u_{1}\right\|_{p}^{p}+C+C_{1}\left\|z_{1}\left(\theta_{t} \omega\right)\right\|_{2}^{2}+\frac{\delta_{1}}{2}\left\|u_{1}\right\|_{p}^{p}+C_{2}\left\|z_{1}\left(\theta_{t} \omega\right)\right\|_{p}^{p} \\
= & -\frac{\delta_{1}}{2}\left\|u_{1}\right\|_{p}^{p}+C+C_{1}\left(\left\|z_{1}\left(\theta_{t} \omega\right)\right\|_{2}^{2}+\left\|z_{1}\left(\theta_{t} \omega\right)\right\|_{p}^{p}\right) .
\end{aligned}
$$


Furthermore, with (2.6) we estimate

$$
\begin{aligned}
I_{2}= & -\int_{D} f\left(x, v_{1}+z_{1}\left(\theta_{t} \omega\right), v_{2}+z_{2}\left(\theta_{t} \omega\right)\right) v_{1} \mathrm{~d} x \\
\leq & \int_{D}\left|f\left(x, v_{1}+z_{1}\left(\theta_{t} \omega\right), v_{2}+z_{2}\left(\theta_{t} \omega\right)\right)\right|\left|u_{1}-z_{1}\left(\theta_{t} \omega\right)\right| \mathrm{d} x \\
\leq & \int_{D} \delta_{4}\left(1+\left|u_{1}\right|^{p_{1}}+\left|u_{2}\right|\right)\left|u_{1}\right| \mathrm{d} x \\
& \quad+\int_{D} \delta_{4}\left(1+\left|u_{1}\right|^{p_{1}}+\left|u_{2}\right|\right)\left|z_{1}\left(\theta_{t} \omega\right)\right| \mathrm{d} x \\
= & \int_{D} \delta_{4}\left(\left|u_{1}\right|+\left|u_{1}\right|^{p_{1}+1}\right) \mathrm{d} x+\int_{D} \delta_{4}\left|u_{1}\right|\left|u_{2}\right| \mathrm{d} x+\delta_{4}|| z_{1}\left(\theta_{t} \omega\right) \|_{1} \\
& \quad+\int_{D} \delta_{4}\left|u_{1}\right|^{p_{1}}\left|z_{1}\left(\theta_{t} \omega\right)\right| \mathrm{d} x+\int_{D} \delta_{4}\left|u_{2}\right|\left|z_{1}\left(\theta_{t} \omega\right)\right| \mathrm{d} x \\
\leq & \int_{D} \delta_{4}\left(\left|u_{1}\right|+\left|u_{1}\right|^{p_{1}+1}\right) \mathrm{d} x+\int_{D} \delta_{4}\left|u_{1}\right|\left|u_{2}\right| \mathrm{d} x+\delta_{4}\left\|z_{1}\left(\theta_{t} \omega\right)\right\|_{2}^{2}+C \\
& \quad+\int_{D} \frac{\delta_{4}}{2}\left|u_{1}\right|^{p_{1}+1} \mathrm{~d} x+C_{1}\left\|z_{1}\left(\theta_{t} \omega\right)\right\|_{p_{1}+1}^{p_{1}+1}+\int_{D} \delta_{4}\left|u_{2}\right|\left|z_{1}\left(\theta_{t} \omega\right)\right| \mathrm{d} x \\
\leq & \int_{D} \delta_{4} \frac{3}{2}\left(\left|u_{1}\right|+\left|u_{1}\right|^{p_{1}+1}\right) \mathrm{d} x+\int_{D} \delta_{4}\left|u_{1}\right|\left|u_{2}\right| \mathrm{d} x+C \\
& +C_{1}\left(\left\|z_{1}\left(\theta_{t} \omega\right)\right\|_{2}^{2}+\left\|z_{1}\left(\theta_{t} \omega\right)\right\|_{p_{1}+1}^{p_{1}+1}\right)+\int_{D} \delta_{4}\left|u_{2}\right|\left|z_{1}\left(\theta_{t} \omega\right)\right| \mathrm{d} x .
\end{aligned}
$$

With (2.9) we compute

$$
\begin{aligned}
I_{3} & =-\int_{D} g\left(x, v_{1}+z_{1}\left(\theta_{t} \omega\right)\right) v_{2} \mathrm{~d} x \\
& \leq \int_{D}\left|g\left(x, u_{1}\right)\right|\left|u_{2}-z_{2}\left(\theta_{t} \omega\right)\right| \mathrm{d} x \\
& \leq \int_{D} \delta_{7}\left(1+\left|u_{1}\right|\right)\left|u_{2}\right| \mathrm{d} x+\int_{D} \delta_{7}\left(1+\left|u_{1}\right|\right)\left|z_{2}\left(\theta_{t} \omega\right)\right| \mathrm{d} x \\
& =\int_{D} \delta_{7}\left(1+\left|u_{1}\right|\right)\left|u_{2}\right| \mathrm{d} x+\delta_{7}\left\|z_{2}\left(\theta_{t} \omega\right)\right\|_{1}+\int_{D} \delta_{7}\left|u_{1}\right|\left|z_{2}\left(\theta_{t} \omega\right)\right| \mathrm{d} x \\
& \leq \int_{D} \delta_{7}\left(1+\left|u_{1}\right|\right)\left|u_{2}\right| \mathrm{d} x+\delta_{7}\left\|z_{2}\left(\theta_{t} \omega\right)\right\|_{2}^{2}+C+\int_{D} \delta_{7}\left|u_{1}\right|\left|z_{2}\left(\theta_{t} \omega\right)\right| \mathrm{d} x .
\end{aligned}
$$


Now, combining the estimates for $I_{2}$ and $I_{3}$ yields

$$
\begin{aligned}
I_{2}+ & I_{3} \\
\leq & \int_{D} \delta_{7}\left(1+\left|u_{1}\right|\right)\left|u_{2}\right| \mathrm{d} x+\int_{D} \delta_{7}\left|u_{1}\right|\left|z_{2}\left(\theta_{t} \omega\right)\right| \mathrm{d} x \\
& +\int_{D} \delta_{4} \frac{3}{2}\left(\left|u_{1}\right|+\left|u_{1}\right|^{p_{1}+1}\right) \mathrm{d} x+\int_{D} \delta_{4}\left|u_{1}\right|\left|u_{2}\right| \mathrm{d} x+\int_{D} \delta_{4}\left|u_{2}\right|\left|z_{1}\left(\theta_{t} \omega\right)\right| \mathrm{d} x \\
& +C+C_{1}\left(\left\|z_{2}\left(\theta_{t} \omega\right)\right\|_{2}^{2}+\left\|z_{1}\left(\theta_{t} \omega\right)\right\|_{2}^{2}+\left\|z_{1}\left(\theta_{t} \omega\right)\right\|_{p_{1}+1}^{p_{1}+1}\right) \\
\leq & \left(\delta_{4}+\delta_{7}\right) \int_{D}\left(1+\left|u_{1}\right|\right)\left|u_{2}\right| \mathrm{d} x+\int_{D} \delta_{7}\left|u_{1} \| z_{2}\left(\theta_{t} \omega\right)\right| \mathrm{d} x \\
& +\int_{D} \delta_{4} \frac{3}{2}\left(\left|u_{1}\right|+\left|u_{1}\right|^{p_{1}+1}\right) \mathrm{d} x+\int_{D} \delta_{4}\left|u_{2}\right|\left|z_{1}\left(\theta_{t} \omega\right)\right| \mathrm{d} x \\
& +C+C_{1}\left(\left\|z_{2}\left(\theta_{t} \omega\right)\right\|_{2}^{2}+\left\|z_{1}\left(\theta_{t} \omega\right)\right\|_{2}^{2}+\left\|z_{1}\left(\theta_{t} \omega\right)\right\|_{p_{1}+1}^{p_{1}+1}\right) \\
\leq & \frac{\delta}{16}\left\|u_{2}\right\|_{2}^{2}+C_{2} \int_{D}\left(1+\left|u_{1}\right|\right)^{2} \mathrm{~d} x+\int_{D} \delta_{7}\left|u_{1}\right|\left|z_{2}\left(\theta_{t} \omega\right)\right| \mathrm{d} x \\
& +\int_{D} \delta_{4} \frac{3}{2}\left(\left|u_{1}\right|+\left|u_{1}\right|^{p_{1}+1}\right) \mathrm{d} x+\int_{D} \delta_{4}\left|u_{2} \| z_{1}\left(\theta_{t} \omega\right)\right| \mathrm{d} x \\
& +C+C_{1}\left(\left\|z_{2}\left(\theta_{t} \omega\right)\right\|_{2}^{2}+\left\|z_{1}\left(\theta_{t} \omega\right)\right\|_{2}^{2}+\left\|z_{1}\left(\theta_{t} \omega\right)\right\|_{p_{1}+1}^{p_{1}+1}\right) \\
= & \frac{\delta}{16}\left\|u_{2}\right\|_{2}^{2}+\delta_{4} \frac{3}{2} \int_{D}\left(\left|u_{1}\right|+\left|u_{1}\right|^{p_{1}+1}+C_{2}\left(1+\left|u_{1}\right|\right)^{2}\right) \mathrm{d} x \\
& +\int_{D} \delta_{7}\left|u_{1}\right|\left|z_{2}\left(\theta_{t} \omega\right)\right| \mathrm{d} x+\int_{D} \delta_{4}\left|u_{2} \| z_{1}\left(\theta_{t} \omega\right)\right| \mathrm{d} x \\
& +C+C_{1}\left(\left\|z_{2}\left(\theta_{t} \omega\right)\right\|_{2}^{2}+\left\|z_{1}\left(\theta_{t} \omega\right)\right\|_{2}^{2}+\left\|z_{1}\left(\theta_{t} \omega\right)\right\|_{p_{1}+1}^{p_{1}+1}\right) \\
\leq & \frac{\delta}{16}\left\|u_{2}\right\|_{2}^{2}+C_{2} \int_{D}\left(1+\left|u_{1}\right|^{q}\right) \mathrm{d} x+\frac{\delta_{1}}{8}\left\|u_{1}\right\|_{2}^{2}+\frac{\delta}{16}\left\|u_{2}\right\|_{2}^{2} \\
& +C+C_{1}\left(\left\|z_{2}\left(\theta_{t} \omega\right)\right\|_{2}^{2}+\left\|z_{1}\left(\theta_{t} \omega\right)\right\|_{2}^{2}+\left\|z_{1}\left(\theta_{t} \omega\right)\right\|_{p_{1}+1}^{p_{1}+1}\right)
\end{aligned}
$$

where we have used that for $q=\max \left\{p_{1}+1,2\right\}<p$ there exists a constant $C_{2}$ such that

$$
C_{1}\left(|\xi|+|\xi|^{p_{1}+1}+C(1+|\xi|)^{2}\right) \leq C_{2}\left(|\xi|^{q}+1\right), \text { for all } \xi \in \mathbb{R} .
$$

Thus,

$$
\begin{aligned}
I_{2}+ & I_{3} \\
\leq & \frac{\delta}{8}\left\|u_{2}\right\|_{2}^{2}+\frac{\delta_{1}}{8}\left\|u_{1}\right\|_{2}^{2}+\frac{\delta_{1}}{4}\left\|u_{1}\right\|_{p}^{p} \\
& +C+C_{1}\left(\left\|z_{2}\left(\theta_{t} \omega\right)\right\|_{2}^{2}+\left\|z_{1}\left(\theta_{t} \omega\right)\right\|_{2}^{2}+\left\|z_{1}\left(\theta_{t} \omega\right)\right\|_{p_{1}+1}^{p_{1}+1}\right) \\
\leq & \frac{\delta}{4}\left\|v_{2}\right\|_{2}^{2}+\frac{\delta_{1} 3}{8}\left\|u_{1}\right\|_{p}^{p}+C \\
& +C_{1}\left(\left\|z_{2}\left(\theta_{t} \omega\right)\right\|_{2}^{2}+\left\|z_{1}\left(\theta_{t} \omega\right)\right\|_{2}^{2}+\left\|z_{1}\left(\theta_{t} \omega\right)\right\|_{p_{1}+1}^{p_{1}+1}\right) .
\end{aligned}
$$


Hence, in total we obtain

$$
\begin{aligned}
\frac{1}{2} \frac{\mathrm{d}}{\mathrm{d} t}( & \left.\left\|v_{1}\right\|_{2}^{2}+\left\|v_{2}\right\|_{2}^{2}\right) \\
\leq & -d\left\|\nabla v_{1}\right\|_{2}^{2}-\frac{\delta_{1}}{2}\left\|u_{1}\right\|_{p}^{p}-\delta\left\|v_{2}\right\|_{2}^{2}+\frac{\delta}{4}\left\|v_{2}\right\|_{2}^{2}+\frac{\delta_{1} 3}{8}\left\|u_{1}\right\|_{p}^{p} \\
& +C+C_{1}\left(\left\|z_{2}\left(\theta_{t} \omega\right)\right\|_{2}^{2}+\left\|z_{1}\left(\theta_{t} \omega\right)\right\|_{2}^{2}+\left\|z_{1}\left(\theta_{t} \omega\right)\right\|_{p_{1}+1}^{p_{1}+1}+\left\|z_{1}\left(\theta_{t} \omega\right)\right\|_{p}^{p}\right) \\
= & -d\left\|\nabla v_{1}\right\|_{2}^{2}-\frac{\delta_{1}}{8}\left\|u_{1}\right\|_{p}^{p}-\frac{3 \delta}{4}\left\|v_{2}\right\|_{2}^{2} \\
& +C+C_{1}\left(\left\|z_{2}\left(\theta_{t} \omega\right)\right\|_{2}^{2}+\left\|z_{1}\left(\theta_{t} \omega\right)\right\|_{2}^{2}+\left\|z_{1}\left(\theta_{t} \omega\right)\right\|_{p_{1}+1}^{p_{1}+1}+\left\|z_{1}\left(\theta_{t} \omega\right)\right\|_{p}^{p}\right) \\
\leq & -\frac{d}{2}\left\|\nabla v_{1}\right\|_{2}^{2}-\frac{d}{2 c}\left\|v_{1}\right\|_{2}^{2}-\frac{3 \delta}{4}\left\|v_{2}\right\|_{2}^{2} \\
& +C+C_{1}\left(\left\|z_{2}\left(\theta_{t} \omega\right)\right\|_{2}^{2}+\left\|z_{1}\left(\theta_{t} \omega\right)\right\|_{p}^{p}\right)
\end{aligned}
$$

and thus

$\frac{\mathrm{d}}{\mathrm{d} t}\left(\left\|v_{1}\right\|_{2}^{2}+\left\|v_{2}\right\|_{2}^{2}\right) \leq-C_{2}\left(\left\|v_{1}\right\|_{2}^{2}+\left\|v_{2}\right\|_{2}^{2}\right)+C+C_{1}\left(\left\|z_{2}\left(\theta_{t} \omega\right)\right\|_{2}^{2}+\left\|z_{1}\left(\theta_{t} \omega\right)\right\|_{p}^{p}\right)$

Now, applying Gronwall's inequality we obtain

$$
\begin{aligned}
\left\|v_{1}\right\|_{2}^{2} & +\left\|v_{2}\right\|_{2}^{2} \\
\leq & \left(\left\|v_{1}^{0}\right\|_{2}^{2}+\left\|v_{2}^{0}\right\|_{2}^{2}\right) \exp \left(-C_{2} t\right)+C_{3}\left(1-\exp \left(-C_{2} t\right)\right) \\
& +C_{1} \int_{0}^{t} \exp \left(-C_{2}(t-s)\right)\left(\left\|z_{2}\left(\theta_{s} \omega\right)\right\|_{2}^{2}+\left\|z_{1}\left(\theta_{s} \omega\right)\right\|_{p}^{p}\right) \mathrm{d} s \\
\leq & \left(\left\|v_{1}^{0}\right\|_{2}^{2}+\left\|v_{2}^{0}\right\|_{2}^{2}\right) \exp \left(-C_{2} t\right)+C_{3} \\
& +C_{1} \int_{0}^{t} \exp \left(-C_{2}(t-s)\right)\left(\left\|z_{2}\left(\theta_{s} \omega\right)\right\|_{2}^{2}+\left\|z_{1}\left(\theta_{s} \omega\right)\right\|_{p}^{p}\right) \mathrm{d} s .
\end{aligned}
$$

We replace $\omega$ by $\theta_{-t} \omega$ (note the $\mathbb{P}$-preserving property of the MDS) and carry out a change of variables

$$
\begin{aligned}
& \left\|v_{1}\left(t, \theta_{-t} \omega, v_{1}^{0}\left(\theta_{-t} \omega\right)\right)\right\|_{2}^{2}+\left\|v_{2}\left(t, \theta_{-t} \omega, v_{2}^{0}\left(\theta_{-t} \omega\right)\right)\right\|_{2}^{2} \\
& \leq\left(\left\|v_{1}^{0}\left(\theta_{-t} \omega\right)\right\|_{2}^{2}+\left\|v_{2}^{0}\left(\theta_{-t} \omega\right)\right\|_{2}^{2}\right) \exp \left(-C_{2} t\right)+C_{3} \\
& \quad+C_{1} \int_{0}^{t} \exp \left(-C_{2}(t-s)\right)\left(\left\|z_{2}\left(\theta_{s-t} \omega\right)\right\|_{2}^{2}+\left\|z_{1}\left(\theta_{s-t} \omega\right)\right\|_{p}^{p}\right) \mathrm{d} s \\
& \leq\left(\left\|v_{1}^{0}\left(\theta_{-t} \omega\right)\right\|_{2}^{2}+\left\|v_{2}^{0}\left(\theta_{-t} \omega\right)\right\|_{2}^{2}\right) \exp \left(-C_{2} t\right)+C_{3} \\
& \quad+C_{1} \int_{-t}^{0} \exp \left(C_{2} s\right)\left(\left\|z_{2}\left(\theta_{s} \omega\right)\right\|_{2}^{2}+\left\|z_{1}\left(\theta_{s} \omega\right)\right\|_{p}^{p}\right) \mathrm{d} s .
\end{aligned}
$$


Now let $\mathcal{D} \in \mathcal{T}$ be arbitrary and $\left(u_{1}^{0}, u_{2}^{0}\right)\left(\theta_{-t} \omega\right) \in \mathcal{D}\left(\theta_{-t} \omega\right)$. Then

$$
\begin{aligned}
\| \varphi( & \left.t, \theta_{-t} \omega,\left(u_{1}^{0}, u_{2}^{0}\right)\left(\theta_{-t} \omega\right)\right) \|_{\mathbb{H}}^{2} \\
= & \left\|v_{1}\left(t, \theta_{-t} \omega, u_{1}^{0}\left(\theta_{-t} \omega\right)-z_{1}\left(\theta_{-t} \omega\right)\right)+z_{1}(\omega)\right\|_{2}^{2} \\
& +\left\|v_{2}\left(t, \theta_{-t} \omega, u_{2}^{0}\left(\theta_{-t} \omega\right)-z_{2}\left(\theta_{-t} \omega\right)\right)+z_{2}(\omega)\right\|_{2}^{2} \\
\leq & 2\left\|v_{1}\left(t, \theta_{-t} \omega, u_{1}^{0}\left(\theta_{-t} \omega\right)-z_{1}\left(\theta_{-t} \omega\right)\right)\right\|_{2}^{2}+2\left\|z_{1}(\omega)\right\|_{2}^{2} \\
& +2\left\|v_{2}\left(t, \theta_{-t} \omega, u_{2}^{0}\left(\theta_{-t} \omega\right)-z_{2}\left(\theta_{-t} \omega\right)\right)\right\|_{2}^{2}+2\left\|z_{2}(\omega)\right\|_{2}^{2} \\
\leq & 2\left(\left\|u_{1}^{0}\left(\theta_{-t} \omega\right)-z_{1}\left(\theta_{-t} \omega\right)\right\|_{2}^{2}+\left\|u_{2}^{0}\left(\theta_{-t} \omega\right)-z_{2}\left(\theta_{-t} \omega\right)\right\|_{2}^{2}\right) \exp \left(-C_{2} t\right) \\
& +2 C_{3}+2 C_{1} \int_{-t}^{0} \exp \left(C_{2} s\right)\left(\left\|z_{2}\left(\theta_{s} \omega\right)\right\|_{2}^{2}+\left\|z_{1}\left(\theta_{s} \omega\right)\right\|_{p}^{p}\right) \mathrm{d} s \\
& +2\left\|z_{1}(\omega)\right\|_{2}^{2}+2\left\|z_{2}(\omega)\right\|_{2}^{2} \\
\leq & 4\left(\left\|u_{1}^{0}\left(\theta_{-t} \omega\right)\right\|_{2}^{2}+\left\|z_{1}\left(\theta_{-t} \omega\right)\right\|_{2}^{2}+\left\|u_{2}^{0}\left(\theta_{-t} \omega\right)\right\|_{2}^{2}+\left\|z_{2}\left(\theta_{-t} \omega\right)\right\|_{2}^{2}\right) \exp \left(-C_{2} t\right) \\
& +2 C_{3}+2 C_{1} \int_{-t}^{0} \exp \left(C_{2} s\right)\left(\left\|z_{2}\left(\theta_{s} \omega\right)\right\|_{2}^{2}+\left\|z_{1}\left(\theta_{s} \omega\right)\right\|_{p}^{p}\right) \mathrm{d} s \\
& +2\left\|z_{1}(\omega)\right\|_{2}^{2}+2\left\|z_{2}(\omega)\right\|_{2}^{2} .
\end{aligned}
$$

Since $\left(u_{1}^{0}, u_{2}^{0}\right)\left(\theta_{-t} \omega\right) \in \mathcal{D}\left(\theta_{-t} \omega\right)$ and since $\left\|z_{1}(\omega)\right\|_{p}^{p}(p \geq 1),\left\|z_{2}(\omega)\right\|_{2}^{2}$ are tempered random variables, we have

$$
\begin{aligned}
\limsup _{t \rightarrow \infty}( & \left\|u_{1}^{0}\left(\theta_{-t} \omega\right)\right\|_{2}^{2}+\left\|z_{1}\left(\theta_{-t} \omega\right)\right\|_{2}^{2} \ldots \\
& \left.+\left\|u_{2}^{0}\left(\theta_{-t} \omega\right)\right\|_{2}^{2}+\left\|z_{2}\left(\theta_{-t} \omega\right)\right\|_{2}^{2}\right) \exp \left(-C_{2} t\right)=0 .
\end{aligned}
$$

Hence,

$$
\begin{aligned}
\limsup _{t \rightarrow \infty}\left\|\varphi\left(t, \theta_{-t} \omega,\left(u_{1}^{0}, u_{2}^{0}\right)\left(\theta_{-t} \omega\right)\right)\right\|_{\mathbb{H}}^{2} \\
\leq 2 C_{3}+2 C_{1} \int_{-\infty}^{0} \exp \left(C_{2} s\right)\left(\left\|z_{2}\left(\theta_{s} \omega\right)\right\|_{2}^{2}+\left\|z_{1}\left(\theta_{s} \omega\right)\right\|_{p}^{p}\right) \mathrm{d} s \\
\quad \quad+2\left\|z_{1}(\omega)\right\|_{2}^{2}+2\left\|z_{2}(\omega)\right\|_{2}^{2} \\
=: \rho(\omega) .
\end{aligned}
$$

Due to the temperedness of $\left\|z_{1}(\omega)\right\|_{p}^{p}$ for $p \geq 1$ and $\left\|z_{2}(\omega)\right\|_{2}^{2}$, the improper integral above exists and $\rho(\omega)>0$ is a $\omega$-dependent constant. As described in Remark 3.6, we can define for some $\epsilon>0$

$$
\mathcal{B}(\omega)=B(0, \rho(\omega)+\epsilon) .
$$

Then $\mathcal{B}=\{\mathcal{B}(\omega)\}_{\omega} \in \mathcal{T}$ is a $\mathcal{T}$-absorbing set for the $\operatorname{RDS} \varphi$ with finite absorption time $t_{\mathcal{T}}(\omega)=\sup _{\mathcal{D} \in \mathcal{T}} t_{\mathcal{D}}(\omega)$.

The random radius $\rho(\omega)$ depends on the restrictions imposed on the nonlinearity and the noise. These were heavily used in Lemma 3.20 in order to derive the expression 3.22 for $\rho(\omega)$. Regarding the structure of $\rho(\omega)$ we infer by Lemma 3.17 that $\rho(\omega)$ is tempered. Although we have now shown the existence of a bounded $\mathcal{T}$-absorbing set for the RDS at hand, we need further steps. To show existence of a random attractor, we would like to make use of 
Theorem 3.11, i.e., we have to show existence of a compact $\mathcal{T}$-absorbing set. This will be the goal of the next subsection.

\subsection{Compact absorbing set}

The classical strategy to find a compact absorbing set in $L^{2}(D)$ for a reactiondiffusion equation is the following: Firstly, one needs to find an absorbing set in $L^{2}(D)$. Secondly, this set is used to find an absorbing set in $H^{1}(D)$ and due to compact embedding this automatically defines a compact absorbing set in $L^{2}(D)$. In our setting the construction of an absorbing set in $H^{1}(D)$ is more complicated as the regularizing effect of the Laplacian is missing in the second component of (3.8). That is solutions with initial conditions in $L^{2}(D)$ will in general only belong to $L^{2}(D)$ and not to $H^{1}(D)$. To overcome this difficulty, we split the solution of the second component into two terms: one which is regular enough, in the sense that it belongs to $H^{1}(D)$ and the another one which asymptotically tends to zero. This splitting method has been used by several authors in the context of partly dissipative systems, see for instance $[20,32]$. Let us now explain the strategy for our setting in more detail. We consider the equations

$$
\frac{\mathrm{d} v_{2}^{1}(t)}{\mathrm{d} t}=-\sigma(x) v_{2}^{1}(t)-g\left(x, v_{1}(t)+z_{1}\left(\theta_{t} \omega\right)\right), v_{2}^{1}(0)=0
$$

and

$$
\frac{\mathrm{d} v_{2}^{2}}{\mathrm{~d} t}=-\sigma(x) v_{2}^{2}, v_{2}^{2}(0)=v_{2}^{0},
$$

then $v_{2}=v_{2}^{1}+v_{2}^{2}$ solves (3.10). Note at this point that we associate the initial condition $v_{2}^{0} \in L^{2}(D)$ to the second part. Now, let $\mathcal{D}=\left(\mathcal{D}_{1}, \mathcal{D}_{2}\right) \in \mathcal{T}$ be arbitrary and $u^{0}=\left(u_{1}^{0}, u_{2}^{0}\right) \in \mathcal{D}$. Then

$$
\begin{aligned}
& \varphi\left(t, \theta_{-t} \omega, u^{0}\left(\theta_{-t} \omega\right)\right) \\
&=\left(u_{1}\left(t, \theta_{-t} \omega, u_{1}^{0}\left(\theta_{-t} \omega\right)\right), u_{2}\left(t, \theta_{-t} \omega, u_{2}^{0}\left(\theta_{-t} \omega\right)\right)\right) \\
&=\left(v_{1}\left(t, \theta_{-t} \omega, v_{1}^{0}\left(\theta_{-t} \omega\right)\right)+z_{1}(\omega),\right. \\
&\left.v_{2}^{1}\left(t, \theta_{-t} \omega, v_{2}^{0}\left(\theta_{-t} \omega\right)\right)+v_{2}^{2}\left(t, \theta_{-t} \omega, v_{2}^{0}\left(\theta_{-t} \omega\right)\right)+z_{2}(\omega)\right) \\
&=\left(v_{1}\left(t, \theta_{-t} \omega, v_{1}^{0}\left(\theta_{-t} \omega\right)\right)+z_{1}(\omega),\right. \\
&\left.v_{2}^{1}\left(t, \theta_{-t} \omega, 0\right)+z_{2}(\omega)\right)+\left(0, v_{2}^{2}\left(t, \theta_{-t} \omega, v_{2}^{0}\left(\theta_{-t} \omega\right)\right)\right) \\
&=: \varphi_{1}\left(t, \theta_{-t} \omega, v_{1}^{0}\left(\theta_{-t} \omega\right)\right)+\varphi_{2}\left(t, \theta_{-t} \omega, v_{2}^{0}\left(\theta_{-t} \omega\right)\right)
\end{aligned}
$$

If we can show that for a certain $t^{*} \geq t_{\mathcal{D}}(\omega)$ there exist tempered random variables $\rho_{1}(\omega), \rho_{2}(\omega)$ such that

$$
\begin{array}{r}
\left\|v_{1}\left(t^{*}, \theta_{-t^{*}} \omega, v_{1}^{0}\left(\theta_{-t^{*}} \omega\right)\right)+z_{1}(\omega)\right\|_{H^{1}(D)}<\rho_{1}(\omega), \\
\left\|v_{2}^{1}\left(t^{*}, \theta_{-t^{*}} \omega, 0\right)+z_{2}(\omega)\right\|_{H^{1}(D)}<\rho_{2}(\omega),
\end{array}
$$

then, because of compact embedding, we know that $\overline{\varphi_{1}\left(t^{*}, \theta_{-t^{*}} \omega, \mathcal{D}_{1}\left(\theta_{-t^{*}} \omega\right)\right)}$ is a compact set in $\mathbb{H}$. If, furthermore

$$
\lim _{t \rightarrow \infty}\left\|v_{2}^{2}\left(t, \theta_{-t} \omega, v_{2}^{0}\left(\theta_{-t} \omega\right)\right)\right\|_{2}=0
$$


then $\varphi_{2}\left(t, \theta_{-t} \omega, \mathcal{D}_{2}\left(\theta_{-t} \omega\right)\right)$ can be regarded as a (random) bounded perturbation and $\overline{\varphi\left(t, \theta_{-t} \omega, \mathcal{D}\left(\theta_{-t} \omega\right)\right)}$ is compact in $\mathbb{H}$ as well, see [28, Theorem 2.1]. Then,

$$
\overline{\varphi\left(t^{*}, \theta_{-t^{*}} \omega, \mathcal{B}\left(\theta_{-t^{*}} \omega\right)\right)}
$$

is a compact absorbing set for the $\operatorname{RDS} \varphi$. We will now prove the necessary estimates (3.25)-(3.27).

Lemma 3.21. Let Assumptions 2.1 and 2.2 hold. Let $\mathcal{D}_{2} \subset L^{2}(D)$ be tempered and $u_{2}^{0} \in \mathcal{D}_{2}$. Then

$$
\lim _{t \rightarrow \infty}\left\|v_{2}^{2}\left(t, \theta_{-t} \omega, v_{2}^{0}\left(\theta_{-t} \omega\right)\right)\right\|_{2}^{2}=0 .
$$

Proof. The solution to (3.24) is given by

$$
v_{2}^{2}(t)=v_{2}^{0} \exp (-\sigma(x) t)
$$

and thus

$$
\begin{aligned}
& \lim _{t \rightarrow \infty}\left\|v_{2}^{2}\left(t, \theta_{-t} \omega, v_{2}^{0}\left(\theta_{-t} \omega\right)\right)\right\|_{2}^{2} \\
& =\lim _{t \rightarrow \infty}\left\|v_{2}^{0}\left(\theta_{-t} \omega\right) \exp (-\sigma(x) t)\right\|_{2}^{2} \\
& \quad \leq \lim _{t \rightarrow \infty}\left\|v_{2}^{0}\left(\theta_{-t} \omega\right)\right\|_{2}^{2} \exp (-\delta t) \\
& \quad \leq \lim _{t \rightarrow \infty}\left(\left\|u_{2}^{0}\left(\theta_{-t} \omega\right)\right\|_{2}^{2}+\left\|z_{2}\left(\theta_{-t} \omega\right)\right\|_{2}^{2}\right) \exp (-\delta t)=0
\end{aligned}
$$

as $u_{2}^{0} \in \mathcal{D}_{2}$ and $\left\|z_{2}(\omega)\right\|_{2}^{2}$ is a tempered random variable.

We now prove boundedness of $v_{1}$ and $v_{2}^{1}$ in $H^{1}(D)$. Therefore we need some auxiliary estimates. First, let us derive uniform estimates for $u_{1} \in L^{p}(D)$ and for $v_{1} \in H^{1}(D)$.

Lemma 3.22. Let Assumptions 2.1 and 2.2 hold. Let $\mathcal{D}_{1} \subset L^{2}(D)$ be tempered and $u_{1}^{0} \in \mathcal{D}_{2}$. Assume $t \geq 0, r>0$, then

$$
\begin{aligned}
& \int_{t}^{t+r}\left\|u_{1}\left(s, \omega, u_{1}^{0}(\omega)\right)\right\|_{p}^{p} \mathrm{~d} s \\
& \quad \leq C r+C_{1} \int_{t}^{t+r}\left(\left\|z_{2}\left(\theta_{s} \omega\right)\right\|_{2}^{2}+\left\|z_{1}\left(\theta_{s} \omega\right)\right\|_{p}^{p}\right) \mathrm{d} s \\
& \quad+\left\|v_{1}\left(t, \omega, v_{1}^{0}(\omega)\right)\right\|_{2}^{2}+\left\|v_{2}\left(t, \omega, v_{2}^{0}(\omega)\right)\right\|_{2}^{2}, \\
& \int_{t}^{t+r}\left\|\nabla v_{1}\left(s, \omega, v_{1}^{0}(\omega)\right)\right\|_{2}^{2} \mathrm{~d} s \\
& \leq C r+C_{1} \int_{t}^{t+r}\left(\left\|z_{2}\left(\theta_{s} \omega\right)\right\|_{2}^{2}+\left\|z_{1}\left(\theta_{s} \omega\right)\right\|_{p}^{p}\right) \mathrm{d} s \\
& \quad+\left\|v_{1}\left(t, \omega, v_{1}^{0}(\omega)\right)\right\|_{2}^{2}+\left\|v_{2}\left(t, \omega, v_{2}^{0}(\omega)\right)\right\|_{2}^{2},
\end{aligned}
$$

where $C, C_{1}$ are deterministic constants. 
Proof. From (3.19) we can derive

$$
\begin{aligned}
& \frac{\mathrm{d}}{\mathrm{d} t}\left(\left\|v_{1}\right\|_{2}^{2}+\left\|v_{2}\right\|_{2}^{2}\right) \\
& \quad \leq-d\left\|\nabla v_{1}\right\|_{2}^{2}-\frac{\delta_{1}}{4}\left\|u_{1}\right\|_{p}^{p}+C+C_{1}\left(\left\|z_{2}\left(\theta_{t} \omega\right)\right\|_{2}^{2}+\left\|z_{1}\left(\theta_{t} \omega\right)\right\|_{p}^{p}\right),
\end{aligned}
$$

and thus by integration

$$
\begin{aligned}
& d \int_{t}^{t+r}\left\|\nabla v_{1}\left(s, \omega, v_{1}^{0}(\omega)\right)\right\|_{2}^{2} \mathrm{~d} s+\frac{\delta_{1}}{4} \int_{t}^{t+r}\left\|u_{1}\left(s, \omega, u_{1}^{0}(\omega)\right)\right\|_{p}^{p} \mathrm{~d} s \\
& \leq C r+C_{1} \int_{t}^{t+r}\left(\left\|z_{2}\left(\theta_{s} \omega\right)\right\|_{2}^{2}+\left\|z_{1}\left(\theta_{s} \omega\right)\right\|_{p}^{p}\right) \mathrm{d} s \\
& \quad+\left\|v_{1}\left(t, \omega, v_{1}^{0}(\omega)\right)\right\|_{2}^{2}+\left\|v_{2}\left(t, \omega, v_{2}^{0}(\omega)\right)\right\|_{2}^{2} .
\end{aligned}
$$

The two statements of the lemma follow directly from this estimate.

Lemma 3.23. Let Assumptions 2.1 and 2.2 hold. Let $\mathcal{D}_{1} \subset L^{2}(D)$ be tempered and $u_{1}^{0} \in \mathcal{D}_{1}$. Assume $t \geq r$, then

$$
\begin{aligned}
& \int_{t}^{t+r}\left\|u_{1}\left(s, \omega, u_{1}^{0}(\omega)\right)\right\|_{2 p-2}^{2 p-2} \mathrm{~d} s \\
& \leq C_{6} r+\int_{t-r}^{t+r} C_{2}\left\|z_{1}\left(\theta_{s} \omega\right)\right\|_{p^{2}-p}^{p^{2}-p}+C_{3}\left\|z_{2}\left(\theta_{s} \omega\right)\right\|_{2}^{2}+C_{4}\left\|v_{2}\left(s, \omega, v_{2}^{0}(\omega)\right)\right\|_{2}^{2} \mathrm{~d} s \\
& \quad+C_{5}\left\|v_{1}\left(t-r, \omega, v_{1}^{0}(\omega)\right)\right\|_{2}^{2}+C_{5}\left\|v_{2}\left(t-r, \omega, v_{2}^{0}(\omega)\right)\right\|_{2}^{2},
\end{aligned}
$$

where $C_{2}, C_{3}, C_{4}, C_{5}, C_{6}$ are deterministic constants. 
Proof. Remember that $v_{1}$ satisfies equation (3.9). Multiplying this equation by $\left|v_{1}\right|^{p-2} v_{1}$ and integrating over $D$ yields

$$
\begin{aligned}
& \frac{1}{p} \frac{\mathrm{d}}{\mathrm{d} t} \int_{D}\left|v_{1}\right|^{p} \mathrm{~d} x \\
& =d \int_{D} \Delta v_{1}(t)\left|v_{1}\right|^{p-2} v_{1} \mathrm{~d} x-\int_{D} h\left(x, v_{1}(t)+z_{1}\left(\theta_{t} \omega\right)\right)\left|v_{1}\right|^{p-2} v_{1} \mathrm{~d} x \\
& -\int_{D} f\left(x, v_{1}(t)+z_{1}\left(\theta_{t} \omega\right), v_{2}(t)+z_{2}\left(\theta_{t} \omega\right)\right)\left|v_{1}\right|^{p-2} v_{1} \mathrm{~d} x \\
& =-d(p-1) \int_{D}\left|\nabla v_{1}\right|^{2}\left|v_{1}\right|^{p-2} \mathrm{~d} x-\int_{D} h\left(x, v_{1}(t)+z_{1}\left(\theta_{t} \omega\right)\right)\left|v_{1}\right|^{p-2} v_{1} \mathrm{~d} x \\
& -\int_{D} f\left(x, v_{1}(t)+z_{1}\left(\theta_{t} \omega\right), v_{2}(t)+z_{2}\left(\theta_{t} \omega\right)\right)\left|v_{1}\right|^{p-2} v_{1} \mathrm{~d} x \\
& \leq-\int_{D}\left(\frac{\delta_{1}}{2^{p}}\left|v_{1}\right|^{p}-C-C_{1}\left(\left|z_{1}\left(\theta_{t} \omega\right)\right|^{2}+\left|z_{1}\left(\theta_{t} \omega\right)\right|^{p}\right)\right)\left|v_{1}\right|^{p-2} \mathrm{~d} x \\
& +\int_{D}\left|f\left(x, v_{1}(t)+z_{1}\left(\theta_{t} \omega\right), v_{2}(t)+z_{2}\left(\theta_{t} \omega\right)\right)\right|\left|v_{1}\right|^{p-2} v_{1} \mathrm{~d} x \\
& \leq-\int_{D} \frac{\delta_{1}}{2^{p}}\left|v_{1}\right|^{2 p-2} \mathrm{~d} x+C \int_{D}\left|v_{1}\right|^{p-2} \mathrm{~d} x \\
& +C_{1} \int_{D}\left(\left|z_{1}\left(\theta_{t} \omega\right)\right|^{2}+\left|z_{1}\left(\theta_{t} \omega\right)\right|^{p}\right)\left|v_{1}\right|^{p-2} \mathrm{~d} x \\
& +\int_{D} \delta_{4}\left(1+\left|v_{1}+z_{1}\left(\theta_{t} \omega\right)\right|^{p_{1}}+\left|v_{2}+z_{2}\left(\theta_{t} \omega\right)\right|\right)\left|v_{1}\right|^{p-2} v_{1} \mathrm{~d} x \\
& \leq-\int_{D} \frac{\delta_{1}}{2^{p}}\left|v_{1}\right|^{2 p-2} \mathrm{~d} x+C \int_{D}\left|v_{1}\right|^{p-2} \mathrm{~d} x+C_{1} \int_{D}\left|v_{1}\right|^{p-1} \mathrm{~d} x \\
& +C_{2} \int_{D}\left(\left|z_{1}\left(\theta_{t} \omega\right)\right|^{2 p-2}+\left|z_{1}\left(\theta_{t} \omega\right)\right|^{p^{2}-p}\right) \mathrm{d} x+\int_{D} \delta_{4}\left(\left|v_{1}\right|^{p-1} \ldots\right. \\
& +C_{3}\left(\left|v_{1}\right|^{p_{1}+p-1}+\left|z_{1}\left(\theta_{t} \omega\right)\right|^{p_{1}}\left|v_{1}\right|^{p-1}+\left|v_{2}\right|\left|v_{1}\right|^{p-1} \ldots\right. \\
& \left.\left.+\left|z_{2}\left(\theta_{t} \omega\right)\right|\left|v_{1}\right|^{p-1}\right)\right) \mathrm{d} x \\
& \leq-\int_{D} \frac{\delta_{1}}{2^{p}}\left|v_{1}\right|^{2 p-2} \mathrm{~d} x+\frac{\delta_{1}}{2^{p} 4} \int_{D}\left|v_{1}\right|^{2 p-2} \mathrm{~d} x+C_{6} \\
& +C_{2} \int_{D}\left(\left|z_{1}\left(\theta_{t} \omega\right)\right|^{2 p-2}+\left|z_{1}\left(\theta_{t} \omega\right)\right|^{p^{2}-p}\right) \mathrm{d} x \\
& +\int_{D} C_{3}\left(\left|z_{1}\left(\theta_{t} \omega\right)\right|^{p_{1}}\left|v_{1}\right|^{p-1}+\left|v_{2}\right|\left|v_{1}\right|^{p-1}+\left|z_{2}\left(\theta_{t} \omega\right)\right|\left|v_{1}\right|^{p-1}\right) \mathrm{d} x,
\end{aligned}
$$

where we have used condition (2.6), the relations $p-1, p-2, p_{1}+p-1<2 p-2$ and the inequality

$$
h\left(x, v_{1}+z_{1}\right) v_{1} \geq \frac{\delta_{1}}{2^{p}}\left|v_{1}\right|^{p}-C-C_{1}\left(\left|z_{1}\right|^{2}+\left|z_{1}\right|^{p}\right),
$$


that can be proved by using conditions (2.5) and (2.10)

$$
\begin{aligned}
h\left(x, v_{1}+z_{1}\right) v_{1} & =h\left(x, v_{1}+z_{1}\right)\left(v_{1}+z_{1}\right)-h\left(x, v_{1}+z_{1}\right) z_{1} \\
& \geq \delta_{1}\left|v_{1}+z_{1}\right|^{p}-\delta_{3}-\left|h\left(x, v_{1}+z_{1}\right)\right|\left|z_{1}\right| \\
& \geq \delta_{1}\left|v_{1}+z_{1}\right|^{p}-\delta_{3}-\left(\delta_{8}+\delta_{8}\left|v_{1}+z_{1}\right|^{p-1}\right)\left|z_{1}\right| \\
& \geq \delta_{1}\left|v_{1}+z_{1}\right|^{p}-C-C_{1}\left|z_{1}\right|^{2}-\delta_{1} / 2\left|v_{1}+z_{1}\right|^{p}-C_{2}\left|z_{1}\right|^{p} \\
& =\frac{\delta_{1}}{2}\left|v_{1}+z_{1}\right|^{p}-C-C_{1}\left(\left|z_{1}\right|^{2}+\left|z_{1}\right|^{p}\right) \\
& \geq\left.\frac{\delta_{1}}{2}|| v_{1}|-| z_{1}\right|^{p}-C-C_{1}\left(\left|z_{1}\right|^{2}+\left|z_{1}\right|^{p}\right) \\
& \geq \frac{\delta_{1}}{2^{p}}\left|v_{1}\right|^{p}-C-C_{1}\left(\left|z_{1}\right|^{2}+\left|z_{1}\right|^{p}\right) .
\end{aligned}
$$

Hence we have

$$
\begin{aligned}
\frac{1}{p} \frac{\mathrm{d}}{\mathrm{d} t} & \int_{D}\left|v_{1}\right|^{p} \mathrm{~d} x+\int_{D} \frac{3}{4} \frac{\delta_{1}}{2^{p}}\left|v_{1}\right|^{2 p-2} \mathrm{~d} x \\
\leq & C_{6}+C_{2} \int_{D}\left(\left|z_{1}\left(\theta_{t} \omega\right)\right|^{2 p-2}+\left|z_{1}\left(\theta_{t} \omega\right)\right|^{p^{2}-p}\right) \mathrm{d} x \\
& +\int_{D} C_{3}\left(\left|z_{1}\left(\theta_{t} \omega\right)\right|^{p_{1}}+\left|v_{2}\right|+\left|z_{2}\left(\theta_{t} \omega\right)\right|\right)\left|v_{1}\right|^{p-1} \mathrm{~d} x \\
\leq & C_{6}+C_{2} \int_{D}\left(\left|z_{1}\left(\theta_{t} \omega\right)\right|^{2 p-2}+\left|z_{1}\left(\theta_{t} \omega\right)\right|^{p^{2}-p}\right) \mathrm{d} x+\int_{D} \frac{1}{4} \frac{\delta_{1}}{2^{p}}\left|v_{1}\right|^{2 p-2} \mathrm{~d} x \\
& +\int_{D} C_{3}\left(\left|z_{1}\left(\theta_{t} \omega\right)\right|^{p_{1}}+\left|v_{2}\right|+\left|z_{2}\left(\theta_{t} \omega\right)\right|\right)^{2} \mathrm{~d} x
\end{aligned}
$$

and thus

$$
\begin{aligned}
\frac{1}{p} \frac{\mathrm{d}}{\mathrm{d} t} \int_{D}\left|v_{1}\right|^{p} \mathrm{~d} x+\int_{D} \frac{1}{2} \frac{\delta_{1}}{2^{p}}\left|v_{1}\right|^{2 p-2} \mathrm{~d} x \\
\leq C_{6}+C_{2} \int_{D}\left(\left|z_{1}\left(\theta_{t} \omega\right)\right|^{2 p-2}+\left|z_{1}\left(\theta_{t} \omega\right)\right|^{p^{2}-p}\right) \mathrm{d} x \\
\quad+\int_{D} C_{3}\left(\left|z_{1}\left(\theta_{t} \omega\right)\right|^{2 p_{1}}+\left|v_{2}(t)\right|^{2}+\left|z_{2}\left(\theta_{t} \omega\right)\right|^{2}\right) \mathrm{d} x .
\end{aligned}
$$

We arrive at the following inequality

$\frac{1}{p} \frac{\mathrm{d}}{\mathrm{d} t}\left\|v_{1}\right\|_{p}^{p}+\frac{\delta_{1}}{2^{p+1}}\left\|v_{1}\right\|_{2 p-2}^{2 p-2} \leq C_{6}+C_{2}\left\|z_{1}\left(\theta_{t} \omega\right)\right\|_{p^{2}-p}^{p^{2}-p}+C_{3}\left\|z_{2}\left(\theta_{t} \omega\right)\right\|_{2}^{2}+C_{3}\left\|v_{2}\right\|_{2}^{2}$

and hence

$$
\frac{\mathrm{d}}{\mathrm{d} t}\left\|v_{1}\right\|_{p}^{p} \leq C_{6}+C_{2}\left\|z_{1}\left(\theta_{t} \omega\right)\right\|_{p^{2}-p}^{p^{2}-p}+C_{3}\left\|z_{2}\left(\theta_{t} \omega\right)\right\|_{2}^{2}+C_{3}\left\|v_{2}\right\|_{2}^{2}-\frac{\delta_{1}}{2^{p+1}}\left\|v_{1}\right\|_{p}^{p} .
$$


With (3.29) we have

$$
\begin{aligned}
\int_{t}^{t+r} & \left\|v_{1}\left(s, \omega, v_{1}^{0}(\omega)\right)\right\|_{p}^{p} \mathrm{~d} s \\
= & \int_{t}^{t+r}\left\|u_{1}\left(s, \omega, v_{1}^{0}(\omega)\right)-z_{1}\left(\theta_{s} \omega\right)\right\|_{p}^{p} \mathrm{~d} s \\
\leq & C r+C_{1} \int_{t}^{t+r}\left(\left\|z_{2}\left(\theta_{s} \omega\right)\right\|_{2}^{2}+\left\|z_{1}\left(\theta_{s} \omega\right)\right\|_{p}^{p}\right) \mathrm{d} s \\
\quad & +C_{2}\left\|v_{1}\left(t, \omega, v_{1}^{0}(\omega)\right)\right\|_{2}^{2}+C_{2}\left\|v_{2}\left(t, \omega, v_{2}^{0}(\omega)\right)\right\|_{2}^{2} .
\end{aligned}
$$

Thus by applying the uniform Gronwall Lemma to (3.34) we have

$$
\begin{aligned}
& \left\|v_{1}\left(t+r, \omega, v_{1}^{0}(\omega)\right)\right\|_{p}^{p} \\
& \leq \quad r C_{6}+\int_{t}^{t+r} C_{2}\left\|z_{1}\left(\theta_{s} \omega\right)\right\|_{p^{2}-p}^{p^{2}-p}+C_{3}\left\|z_{2}\left(\theta_{s} \omega\right)\right\|_{2}^{2}+C_{4}\left\|v_{2}\left(s, \omega, v_{2}^{0}(\omega)\right)\right\|_{2}^{2} \mathrm{~d} s \\
& \quad+C_{5}\left\|v_{1}\left(t, \omega, v_{1}^{0}(\omega)\right)\right\|_{2}^{2}+C_{5}\left\|v_{2}\left(t, \omega, v_{2}^{0}(\omega)\right)\right\|_{2}^{2} .
\end{aligned}
$$

Now integrating (3.33) between $t$ and $t+r$ yields

$$
\begin{aligned}
& \int_{t}^{t+r}\left\|v_{1}\left(s, \omega, v_{1}(\omega)\right)\right\|_{2 p-2}^{2 p-2} \mathrm{~d} s \\
& \quad \leq C_{6} r+\int_{t}^{t+r} C_{2}\left\|z_{1}\left(\theta_{s} \omega\right)\right\|_{p^{2}-p}^{p^{2}-p}+C_{3}\left\|z_{2}\left(\theta_{s} \omega\right)\right\|_{2}^{2}+C_{3}\left\|v_{2}\left(s, \omega, v_{2}^{0}(\omega)\right)\right\|_{2}^{2} \mathrm{~d} s \\
& \quad+C\left\|v_{1}\left(t, \omega, v_{1}^{0}(\omega)\right)\right\|_{p}^{p}
\end{aligned}
$$

and thus for $t \geq r$ using (3.35)

$$
\begin{aligned}
& \int_{t}^{t+r} \quad\left\|v_{1}\left(s, \omega, v_{1}(\omega)\right)\right\|_{2 p-2}^{2 p-2} \mathrm{~d} s \\
& \quad \leq C_{6} r+\int_{t-r}^{t+r} C_{2}\left\|z_{1}\left(\theta_{s} \omega\right)\right\|_{p^{2}-p}^{p^{2}-p}+C_{3}\left\|z_{2}\left(\theta_{s} \omega\right)\right\|_{2}^{2}+C_{4}\left\|v_{2}\left(s, \omega, v_{2}^{0}(\omega)\right)\right\|_{2}^{2} \mathrm{~d} s \\
& \quad+C_{5}\left\|v_{1}\left(t-r, \omega, v_{1}^{0}(\omega)\right)\right\|_{2}^{2}+C_{5}\left\|v_{2}\left(t-r, \omega, v_{2}^{0}(\omega)\right)\right\|_{2}^{2} .
\end{aligned}
$$

In total this leads to

$$
\begin{aligned}
& \int_{t}^{t+r}\left\|u_{1}\left(s, \omega, v_{1}(\omega)\right)\right\|_{2 p-2}^{2 p-2} \mathrm{~d} s \\
& \leq C_{6} r+\int_{t-r}^{t+r} C_{2}\left\|z_{1}\left(\theta_{s} \omega\right)\right\|_{p^{2}-p}^{p^{2}-p}+C_{3}\left\|z_{2}\left(\theta_{s} \omega\right)\right\|_{2}^{2}+C_{4}\left\|v_{2}\left(s, \omega, v_{2}^{0}(\omega)\right)\right\|_{2}^{2} \mathrm{~d} s \\
& \quad+C_{5}\left\|v_{1}\left(t-r, \omega, v_{1}^{0}(\omega)\right)\right\|_{2}^{2}+C_{5}\left\|v_{2}\left(t-r, \omega, v_{2}^{0}(\omega)\right)\right\|_{2}^{2} \\
& \quad+\int_{t}^{t+r}\left\|z_{1}\left(\theta_{s} \omega\right)\right\|_{2 p-2}^{2 p-2} \mathrm{~d} s \\
& \quad C_{6} r+\int_{t-r}^{t+r} C_{2}\left\|z_{1}\left(\theta_{s} \omega\right)\right\|_{p^{2}-p}^{p^{2}-p}+C_{3}\left\|z_{2}\left(\theta_{s} \omega\right)\right\|_{2}^{2}+C_{4}\left\|v_{2}\left(s, \omega, v_{2}^{0}(\omega)\right)\right\|_{2}^{2} \mathrm{~d} s \\
& \quad+C_{5}\left\|v_{1}\left(t-r, \omega, v_{1}^{0}(\omega)\right)\right\|_{2}^{2}+C_{5}\left\|v_{2}\left(t-r, \omega, v_{2}^{0}(\omega)\right)\right\|_{2}^{2},
\end{aligned}
$$

and this finishes the proof. 
One can also use appropriate shifts within the integrals on the left hand sides in (3.29), (3.30), (3.31) to obtain simpler forms of the $\omega$-dependent constants on the right hand side, see for instance [33, Lemma 4.3, 4.4]. More precisely, in case of (3.29) one can for instance obtain an estimate of the form

$$
\int_{t}^{t+r}\left\|u_{1}\left(s, \theta_{-t-r} \omega, u_{1}^{0}\left(\theta_{-t-r} \omega\right)\right)\right\|_{p}^{p} \leq c(1+\widetilde{\rho}(\omega))
$$

where $\tilde{\rho}(\omega)$ is a random constant. Nevertheless such estimates hold for every $\omega$, independent of the shift that one inserts inside the integral on the left hand side. Without the appropriate shifts on the left hand sides, as in the lemmas above, the constants on the right hand sides depend on the shift. Next, we are going to show the boundedness of $v_{1}$ in $H^{1}(D)$.

Lemma 3.24. Let Assumptions 2.1 and 2.2 hold. Let $\mathcal{D}=\left(\mathcal{D}_{1}, \mathcal{D}_{2}\right) \in \mathcal{T}$ and $u^{0} \in \mathcal{D}$. Assume $t \geq t_{\mathcal{D}}(\omega)+2 r$ for some $r>0$ then

$$
\left\|\nabla v_{1}\left(t, \theta_{-t} \omega, v_{1}^{0}\left(\theta_{-t} \omega\right)\right)\right\|_{2}^{2} \leq \rho_{1}(\omega)
$$

where $\rho_{1}(\omega)$ is a tempered random variable.

Proof. Remember that $v_{1}$ satisfies the equation (3.9) and thus

$$
\begin{aligned}
& \frac{1}{2} \frac{\mathrm{d}}{\mathrm{d} t}\left\|\nabla v_{1}\right\|_{2}^{2}=\left\langle\frac{\mathrm{d}}{\mathrm{d} t} v_{1},-\Delta v_{1}\right\rangle \\
&=\left\langle d \Delta v_{1}-h\left(x, v_{1}+z_{1}\left(\theta_{t} \omega\right)\right)-f\left(x, v_{1}+z_{1}\left(\theta_{t} \omega\right), v_{2}+z_{2}\left(\theta_{t} \omega\right)\right),-\Delta v_{1}\right\rangle \\
&=-d\left\|\Delta v_{1}\right\|_{2}^{2}+\left\langle h\left(x, v_{1}+z_{1}\left(\theta_{t} \omega\right)\right), \Delta v_{1}\right\rangle \\
& \quad+\left\langle f\left(x, v_{1}+z_{1}\left(\theta_{t} \omega\right), v_{2}+z_{2}\left(\theta_{t} \omega\right)\right), \Delta v_{1}\right\rangle \\
& \leq- d\left\|\Delta v_{1}\right\|_{2}^{2}+\int_{D} \delta_{8}\left(1+\left|u_{1}\right|^{p-1}\right)\left|\Delta v_{1}\right| \mathrm{d} x \\
&+\int_{D} \delta_{4}\left(1+\left|u_{1}\right|^{p_{1}}+\left|u_{2}\right|\right)\left|\Delta v_{1}\right| \mathrm{d} x \\
& \leq-d\left\|\Delta v_{1}\right\|_{2}^{2}+C \int_{D}\left(2+\left|u_{1}\right|^{p-1}+\left|u_{1}\right|^{p_{1}}+\left|u_{2}\right|\right)\left|\Delta v_{1}\right| \mathrm{d} x \\
& \leq-\frac{d}{2}\left\|\Delta v_{1}\right\|_{2}^{2}+C \int_{D}\left(1+\left|u_{1}\right|^{p-1}+\left|u_{1}\right|^{p_{1}}+\left|u_{2}\right|\right)^{2} \mathrm{~d} x \\
& \leq-\frac{d}{2}\left\|\Delta v_{1}\right\|_{2}^{2}+C \int_{D}\left(1+\left|u_{1}\right|^{2 p-2}+\left|u_{2}\right|^{2}\right) \mathrm{d} x \\
&=-\frac{d}{2}\left\|\Delta v_{1}\right\|_{2}^{2}+C_{1}+C\left\|u_{1}\right\|_{2 p-2}^{2 p-2}+C\left\|u_{2}\right\|_{2}^{2} \\
& \leq-\frac{d c}{2}\left\|\nabla v_{1}\right\|_{2}^{2}+C_{1}+C\left\|u_{1}\right\|_{2 p-2}^{2 p-2}+C\left\|_{2}\right\|_{2}^{2} .
\end{aligned}
$$


We want to apply the uniform Gronwall Lemma now. Therefore, note

$$
\begin{aligned}
\frac{\mathrm{d}}{\mathrm{d} t} \underbrace{\left\|\nabla v_{1}\left(t, \omega, v_{1}^{0}(\omega)\right)\right\|_{2}^{2}}_{:=y(t)} \leq & \underbrace{-d c}_{:=g(t)}\left\|\nabla v_{1}\left(t, \omega, v_{1}^{0}(\omega)\right)\right\|_{2}^{2} \\
& +\underbrace{C_{1}+C\left\|u_{1}\left(t, \omega, u_{1}^{0}(\omega)\right)\right\|_{2 p-2}^{2 p-2}+C\left\|u_{2}\left(t, \omega, u_{2}^{0}(\omega)\right)\right\|_{2}^{2}}_{:=h(t)} .
\end{aligned}
$$

We calculate

$$
\int_{t}^{t+r} g(s) \mathrm{d} s \leq 0
$$

and

$$
\begin{aligned}
& \int_{t}^{t+r}\left\|\nabla v_{1}\left(s, \omega, v_{1}^{0}(\omega)\right)\right\|_{2}^{2} \mathrm{~d} s \\
& \leq C r+C_{1} \int_{t}^{t+r}\left(\left\|z_{2}\left(\theta_{s} \omega\right)\right\|_{2}^{2}+\left\|z_{1}\left(\theta_{s} \omega\right)\right\|_{p}^{p}\right) \mathrm{d} s \\
& \quad+C_{2}\left(\left\|v_{1}\left(t, \omega, v_{1}^{0}(\omega)\right)\right\|_{2}^{2}+\left\|v_{2}\left(t, \omega, v_{2}^{0}(\omega)\right)\right\|_{2}^{2}\right.
\end{aligned}
$$

where we have applied Lemma 3.22. By Lemma 3.23 for $t \geq r$

$$
\begin{aligned}
& \int_{t}^{t+r} \quad\left\|u_{1}\left(s, \omega, u_{1}^{0}(\omega)\right)\right\|_{2 p-2}^{2 p-2} \mathrm{~d} s \\
& \quad \leq C_{6} r+\int_{t-r}^{t+r} C_{2}\left\|z_{1}\left(\theta_{s} \omega\right)\right\|_{p^{2}-p}^{p^{2}-p}+C_{3}\left\|z_{2}\left(\theta_{s} \omega\right)\right\|_{2}^{2}+C_{4}\left\|u_{2}\left(s, \omega, v_{2}^{0}(\omega)\right)\right\|_{2}^{2} \mathrm{~d} s \\
& \quad+C_{5}\left\|v_{1}\left(t-r, \omega, v_{1}^{0}(\omega)\right)\right\|_{2}^{2}+C_{5}\left\|v_{2}\left(t-r, \omega, v_{2}^{0}(\omega)\right)\right\|_{2}^{2} .
\end{aligned}
$$

Now, the uniform Gronwall Lemma yields for $t \geq r$

$$
\begin{aligned}
\left\|\nabla v_{1}\left(t+r, \omega, v_{1}^{0}(\omega)\right)\right\|_{2}^{2} \\
\leq C+C_{1} \int_{t}^{t+r}\left(\left\|z_{2}\left(\theta_{s} \omega\right)\right\|_{2}^{2}+\left\|z_{1}\left(\theta_{s} \omega\right)\right\|_{p}^{p}\right) \mathrm{d} s \\
\quad+C_{2}\left(\left\|v_{1}\left(t, \omega, v_{1}^{0}(\omega)\right)\right\|_{2}^{2}+\left\|v_{2}\left(t, \omega, v_{2}^{0}(\omega)\right)\right\|_{2}^{2}\right) \\
\quad+C_{3} \int_{t-r}^{t+r}\left\|z_{1}\left(\theta_{s} \omega\right)\right\|_{p^{2}-p}^{p^{2}-p}+\left\|z_{2}\left(\theta_{s} \omega\right)\right\|_{2}^{2}+\left\|u_{2}\left(s, \omega, v_{2}^{0}(\omega)\right)\right\|_{2}^{2} \mathrm{~d} s \\
+C_{4}\left(\left\|v_{1}\left(t-r, \omega, v_{1}^{0}(\omega)\right)\right\|_{2}^{2}+\left\|v_{2}\left(t-r, \omega, v_{2}^{0}(\omega)\right)\right\|_{2}^{2}\right) \\
+C_{5} \int_{t}^{t+r}\left\|u_{2}\left(s, \omega, u_{2}^{0}(\omega)\right)\right\|_{2}^{2} \mathrm{~d} s \\
\leq \quad+C_{1} \int_{t-r}^{t+r}\left\|u_{2}\left(s, \omega, u_{2}^{0}(\omega)\right)\right\|_{2}^{2} \mathrm{~d} s \\
\quad+C_{2}^{t+r}\left\|z_{t-r}\left(\theta_{s} \omega\right)\right\|_{p^{2}-p}^{p^{2}-p}+\left\|z_{2}\left(\theta_{s} \omega\right)\right\|_{2}^{2} \mathrm{~d} s \\
\quad+C_{3}\left(\left\|v_{1}\left(t, \omega, v_{1}^{0}(\omega)\right)\right\|_{2}^{2}+\left\|v_{2}\left(t, \omega, v_{2}^{0}(\omega)\right)\right\|_{2}^{2} \ldots\right. \\
\left.\quad+\left\|v_{1}\left(t-r, \omega, v_{1}^{0}(\omega)\right)\right\|_{2}^{2}+\left\|v_{2}\left(t-r, \omega, v_{2}^{0}(\omega)\right)\right\|_{2}^{2}\right) .
\end{aligned}
$$


That is, for $t \geq 0$ we have

$$
\begin{aligned}
& \left\|\nabla v_{1}\left(t+2 r, \omega, v_{1}^{0}(\omega)\right)\right\|_{2}^{2} \\
& \leq C+C_{1} \int_{t}^{t+2 r}\left\|v_{2}\left(s, \omega, u_{2}^{0}(\omega)\right)\right\|_{2}^{2} \mathrm{~d} s \\
& \quad+C_{2} \int_{t}^{t+2 r}\left\|z_{1}\left(\theta_{s} \omega\right)\right\|_{p^{2}-p}^{p^{2}-p}+\left\|z_{2}\left(\theta_{s} \omega\right)\right\|_{2}^{2} \mathrm{~d} s \\
& \quad+C_{3}\left(\left\|v_{1}\left(t+r, \omega, v_{1}^{0}(\omega)\right)\right\|_{2}^{2}+\left\|v_{2}\left(t+r, \omega, v_{2}^{0}(\omega)\right)\right\|_{2}^{2} \ldots\right. \\
& \left.\quad+\left\|v_{1}\left(t, \omega, v_{1}^{0}(\omega)\right)\right\|_{2}^{2}+\left\|v_{2}\left(t, \omega, v_{2}^{0}(\omega)\right)\right\|_{2}^{2}\right) .
\end{aligned}
$$

Let us recall that our goal is to find a $t^{*} \geq t_{\mathcal{D}}(\omega)$ such that (3.25) holds. Now assume that $t \geq t_{\mathcal{D}}(\omega)$. We replace $\omega$ by $\theta_{-t-2 r} \omega$ (again note the $\mathbb{P}$-preserving property of the MDS), then

$$
\begin{aligned}
& \left\|\nabla v_{1}\left(t+2 r, \theta_{-t-2 r} \omega, v_{1}^{0}\left(\theta_{-t-2 r} \omega\right)\right)\right\|_{2}^{2} \\
& \leq C+C_{1} \int_{t}^{t+2 r}\left\|v_{2}\left(s, \theta_{-t-2 r} \omega, u_{2}^{0}\left(\theta_{-t-2 r} \omega\right)\right)\right\|_{2}^{2} \mathrm{~d} s \\
& +C_{2} \int_{t}^{t+2 r}\left\|z_{1}\left(\theta_{s-t-2 r} \omega\right)\right\|_{p^{2}-p}^{p^{2}-p}+\left\|z_{2}\left(\theta_{s-t-2 r} \omega\right)\right\|_{2}^{2} \mathrm{~d} s \\
& +C_{3}\left(\left\|v_{1}\left(t+r, \theta_{-t-2 r} \omega, v_{1}^{0}\left(\theta_{-t-2 r} \omega\right)\right)\right\|_{2}^{2} \ldots\right. \\
& \quad+\left\|v_{2}\left(t+r, \theta_{-t-2 r} \omega, v_{2}^{0}\left(\theta_{-t-2 r} \omega\right)\right)\right\|_{2}^{2} \ldots \\
& \quad+\left\|v_{1}\left(t, \theta_{-t-2 r} \omega, v_{1}^{0}\left(\theta_{-t-2 r} \omega\right)\right)\right\|_{2}^{2} \ldots \\
& \left.\quad+\left\|v_{2}\left(t, \theta_{-t-2 r} \omega, v_{2}^{0}\left(\theta_{-t-2 r} \omega\right)\right)\right\|_{2}^{2}\right) .
\end{aligned}
$$

As $t \geq t_{\mathcal{D}}(\omega)$ we know by the absorption property that there exists a $\tilde{\rho}(\omega)$ such that

$$
\left\|v_{1}\left(t, \theta_{-t} \omega, v_{1}^{0}\left(\theta_{-t} \omega\right)\right)\right\|_{2}^{2} \leq \tilde{\rho}(\omega)
$$

and thus replacing $\omega$ by $\theta_{-2 r} \omega$

$$
\left\|v_{1}\left(t, \theta_{-t-2 r} \omega, v_{1}^{0}\left(\theta_{-t-2 r} \omega\right)\right)\right\|_{2}^{2} \leq \tilde{\rho}\left(\theta_{-2 r} \omega\right) .
$$

Similarly, we know that

$$
\left\|v_{1}\left(t+r, \theta_{-t-r} \omega, v_{1}^{0}\left(\theta_{-t-r} \omega\right)\right)\right\|_{2}^{2} \leq \tilde{\rho}\left(\theta_{-r} \omega\right),
$$

and thus by replacing $\omega$ by $\theta_{-r} \omega$

$$
\left\|v_{1}\left(t+r, \theta_{-t-2 r} \omega, v_{1}^{0}\left(\theta_{-t-2 r} \omega\right)\right)\right\|_{2}^{2} \leq \tilde{\rho}\left(\theta_{-2 r} \omega\right) .
$$

The same arguments hold for $v_{2}$. Furthermore, as $t \geq t_{\mathcal{D}}(\omega)$ and we know from Lemma 3.20 that there exists a tempered random variable $\hat{\rho}(\omega)$ such that for $s \in(t, t+2 r)$

$$
\left\|v_{2}\left(s, \theta_{-s} \omega, u_{2}^{0}\left(\theta_{-s} \omega\right)\right)\right\|_{2}^{2} \leq \hat{\rho}(\omega)
$$


and thus

$$
\begin{aligned}
& \int_{t}^{t+2 r}\left\|v_{2}\left(s, \theta_{-t-2 r} \omega, u_{2}^{0}\left(\theta_{-t-2 r} \omega\right)\right)\right\|_{2}^{2} \mathrm{~d} s \\
& \quad \leq \int_{t}^{t+2 r} \hat{\rho}\left(\theta_{s-t-2 r} \omega\right) \mathrm{d} s=\int_{0}^{2 r} \hat{\rho}\left(\theta_{\tau-2 r} \omega\right) \mathrm{d} \tau=\int_{-2 r}^{0} \hat{\rho}\left(\theta_{y} \omega\right) \mathrm{d} y .
\end{aligned}
$$

With similar substitutions in the integral over $\left\|z_{1}\left(\theta_{s-t-2 r} \omega\right)\right\|_{p^{2}-p}^{p^{2}-p}$ and $\left\|z_{2}\left(\theta_{s-t-2 r} \omega\right)\right\|_{2}^{2}$ we arrive at

$$
\begin{aligned}
& \left\|\nabla v_{1}\left(t+2 r, \theta_{-t-2 r} \omega, v_{1}^{0}\left(\theta_{-t-2 r} \omega\right)\right)\right\|_{2}^{2} \\
& \leq C+C_{1} \int_{-2 r}^{0} \hat{\rho}\left(\theta_{y} \omega\right) \mathrm{d} y+C_{2} \int_{-2 r}^{0}\left\|z_{1}\left(\theta_{y} \omega\right)\right\|_{p^{2}-p}^{p^{2}-p}+\left\|z_{2}\left(\theta_{y} \omega\right)\right\|_{2}^{2} \mathrm{~d} y \\
& \quad+C_{3} \tilde{\rho}\left(\theta_{-2 r} \omega\right),
\end{aligned}
$$

where the right hand side is independent of $t$. Due to the temperedness of all terms involved, they can be combined into one tempered random variable $\rho_{1}(\omega)$ such that for $t \geq t_{\mathcal{D}}(\omega)+2 r=: t^{*}$ we have

$$
\left\|\nabla v_{1}\left(t, \theta_{-t} \omega, v_{1}^{0}\left(\theta_{-t} \omega\right)\right)\right\|_{2}^{2} \leq \rho_{1}(\omega)
$$

this concludes the proof. $H^{1}(D)$.

We are now able to prove the boundedness of the first term of $v_{2}$ in

Lemma 3.25. Let Assumptions 2.1 and 2.2 hold. Let $\mathcal{D}=\left(\mathcal{D}_{1}, \mathcal{D}_{2}\right) \in \mathcal{T}$ and $u^{0} \in \mathcal{D}$. Assume $t \geq t_{\mathcal{D}}(\omega)+2 r$ for some $r>0$. Then we have

$$
\left\|\nabla v_{2}^{1}\left(t, \theta_{-t} \omega, 0\right)\right\|_{2}^{2} \leq \rho_{2}(\omega)
$$

where $\rho_{2}(\omega)$ is a tempered random variable.

Proof. Remember that $v_{2}^{1}$ satisfies the equation (3.23) and thus

$$
\begin{aligned}
\frac{1}{2} \frac{\mathrm{d}}{\mathrm{d} t}\left\|\nabla v_{2}^{1}\right\|_{2}^{2} & =\left\langle\frac{\mathrm{d}}{\mathrm{d} t} v_{2}^{1},-\Delta v_{2}^{1}\right\rangle \\
& =\left\langle-\sigma(x) v_{2}^{1}-g\left(x, v_{1}+z_{1}\right),-\Delta v_{2}^{1}\right\rangle \\
& =\underbrace{\left\langle\sigma(x) v_{2}^{1}, \Delta v_{2}^{1}\right\rangle}_{=: L_{1}}+\underbrace{\left\langle g\left(x, v_{1}+z_{1}\right), \Delta v_{2}^{1}\right\rangle}_{=: L_{2}} .
\end{aligned}
$$

We estimate $L_{1}$ and $L_{2}$ separately

$$
\begin{aligned}
L_{1} & =\int_{D} \sigma(x) v_{2}^{1} \Delta v_{2}^{1} \mathrm{~d} x \\
& =-\int_{D} \nabla\left(\sigma(x) v_{2}^{1}\right) \cdot \nabla v_{2}^{1} \mathrm{~d} x \\
& \leq-\delta\left\|\nabla v_{2}^{1}\right\|_{2}^{2}-\int_{D} \nabla \sigma(x) v_{2}^{1} \cdot \nabla v_{2}^{1} \mathrm{~d} x
\end{aligned}
$$


and

$$
\begin{aligned}
L_{2} & =\int_{D} g\left(x, v_{1}+z_{1}\right) \Delta v_{2}^{1} \mathrm{~d} x=-\int_{D} \nabla g\left(x, v_{1}+z_{1}\right) \cdot \nabla v_{2}^{1} \mathrm{~d} x \\
& =-\int_{D}\left(\nabla g\left(x, v_{1}+z_{1}\right)+\partial_{\xi} g\left(x, v_{1}+z_{1}\right) \nabla\left(v_{1}+z_{1}\right)\right) \cdot \nabla v_{2}^{1} \mathrm{~d} x
\end{aligned}
$$

where in the last equation the gradient is to be understood as

$$
\nabla g\left(x, v_{1}+z_{1}\right)=\left(\partial_{x_{1}} g\left(x, v_{1}+z_{1}\right), \ldots, \partial_{x_{n}} g\left(x, v_{1}+z_{1}\right)\right)^{\top} .
$$

Hence,

$$
\begin{aligned}
& \frac{\mathrm{d}}{\mathrm{d} t}\left\|\nabla v_{2}^{1}\right\|_{2}^{2}+2 \delta\left\|\nabla v_{2}^{1}\right\|_{2}^{2} \\
& \leq 2 \int_{D}\left|\nabla \sigma(x) v_{2}^{1}+\nabla g\left(x, v_{1}+z_{1}\right)+\partial_{\xi} g\left(x, v_{1}+z_{1}\right) \nabla\left(v_{1}+z_{1}\right)\right|\left|\nabla v_{2}^{1}\right| \mathrm{d} x \\
& \leq \frac{1}{\delta} \int_{D}\left|\nabla \sigma(x) v_{2}^{1}+\nabla g\left(x, v_{1}+z_{1}\right)+\partial_{\xi} g\left(x, v_{1}+z_{1}\right) \nabla\left(v_{1}+z_{1}\right)\right|^{2} \mathrm{~d} x \\
& \quad+\delta\left\|\nabla v_{2}^{1}\right\|_{2}^{2}
\end{aligned}
$$

and further with $(2.8)$

$$
\begin{aligned}
\frac{\mathrm{d}}{\mathrm{d} t} \| & \nabla v_{2}^{1}\left\|_{2}^{2}+\delta\right\| \nabla v_{2}^{1} \|_{2}^{2} \\
\leq & \frac{1}{\delta} \int_{D} \sum_{i=1}^{n}\left(\left|\partial_{x_{i}} \sigma(x) v_{2}^{1}\right|+\left|\partial_{x_{i}} g\left(x, v_{1}+z_{1}\right)\right| \ldots\right. \\
& \left.+\left|\partial_{\xi} g\left(x, v_{1}+z_{1}\right) \partial_{x_{i}}\left(v_{1}+z_{1}\right)\right|\right)^{2} \mathrm{~d} x \\
\leq & \frac{1}{\delta} \int_{D} \sum_{i=1}^{n}\left(C\left|v_{2}^{1}\right|+\delta_{5}\left(1+\left|v_{1}+z_{1}\right|\right)+\delta_{5}\left|\partial_{x_{i}}\left(v_{1}+z_{1}\right)\right|\right)^{2} \mathrm{~d} x \\
\leq & \frac{2}{\delta}\left(C+\delta_{5}\right)^{2} n \int_{D}\left(\left|v_{2}^{1}\right|+1+\left|v_{1}+z_{1}\right|\right)^{2} \mathrm{~d} x+\frac{2 \delta_{5}^{2}}{\delta} \int_{D} \sum_{i=1}^{n}\left|\partial_{x_{i}}\left(v_{1}+z_{1}\right)\right|^{2} \mathrm{~d} x \\
= & \frac{2}{\delta}\left(C+\delta_{5}\right)^{2} n \int_{D}\left(\left|v_{2}^{1}\right|+1+\left|v_{1}+z_{1}\right|\right)^{2} \mathrm{~d} x+\frac{2 \delta_{5}^{2}}{\delta}\left\|\nabla\left(v_{1}+z_{1}\right)\right\|_{2}^{2} \\
\leq & C_{1}+C_{2}\left(\left\|v_{2}^{1}\right\|_{2}^{2}+\left\|v_{1}\right\|_{2}^{2}+\left\|z_{1}\right\|_{2}^{2}\right)+C_{3}\left(\left\|\nabla v_{1}\right\|_{2}^{2}+\left\|\nabla z_{1}\right\|_{2}^{2}\right) .
\end{aligned}
$$

where $C:=\max _{1 \leq i \leq n} \max _{x \in \bar{D}}\left|\partial_{x_{i}} \sigma(x)\right|$. Next, we apply Gronwall's inequality while taking the initial condition into account and we obtain for $t \geq 0$

$$
\begin{aligned}
\left\|\nabla v_{2}^{1}\right\|_{2}^{2} \leq \int_{0}^{t}\left[C_{1}+C_{2}\left(\left\|v_{2}^{1}\right\|_{2}^{2}+\left\|v_{1}\right\|_{2}^{2}+\left\|z_{1}\right\|_{2}^{2}\right)+C_{3}\left(\left\|\nabla v_{1}\right\|_{2}^{2}+\left\|\nabla z_{1}\right\|_{2}^{2}\right)\right] \ldots \\
\quad \times \exp ((s-t) \delta) \mathrm{d} s .
\end{aligned}
$$

We have from (3.19) the following equation $\frac{\mathrm{d}}{\mathrm{d} t}\left(\left\|v_{1}\right\|_{2}^{2}+\left\|v_{2}\right\|_{2}^{2}\right)+M\left(\left\|v_{1}\right\|_{2}^{2}+\left\|v_{2}\right\|_{2}^{2}\right)+d\left\|\nabla v_{1}\right\|_{2}^{2} \leq \hat{C}+\tilde{C}\left(\left\|z_{2}\left(\theta_{t} \omega\right)\right\|_{2}^{2}+\left\|z_{1}\left(\theta_{t} \omega\right)\right\|_{p}^{p}\right)$, 
where $M=\min \{d / c, \delta\}$ and certain constants $\hat{C}, \tilde{C}$. We multiply (3.40) by $\exp (M t)$ and integrate between 0 and $t$

$$
\begin{aligned}
\int_{0}^{t} & \exp (M s) \frac{\mathrm{d}}{\mathrm{d} s}\left(\left\|v_{1}\right\|_{2}^{2}+\left\|v_{2}\right\|_{2}^{2}\right) \mathrm{d} s+M \int_{0}^{t} \exp (M s)\left(\left\|v_{1}\right\|_{2}^{2}+\left\|v_{2}\right\|_{2}^{2}\right) \mathrm{d} s \\
& +d \int_{0}^{t} \exp (M s)\left\|\nabla v_{1}\right\|_{2}^{2} \mathrm{~d} s \\
\leq & \int_{0}^{t} \hat{C} \exp (M s) \mathrm{d} s+\tilde{C} \int_{0}^{t} \exp (M s)\left(\left\|z_{2}\left(\theta_{s} \omega\right)\right\|_{2}^{2}+\left\|z_{1}\left(\theta_{s} \omega\right)\right\|_{p}^{p}\right) \mathrm{d} s .
\end{aligned}
$$

This yields

$$
\begin{aligned}
& \int_{0}^{t} \exp (M(s-t))\left\|\nabla v_{1}\left(s, \omega, v_{1}^{0}(\omega)\right)\right\|_{2}^{2} \mathrm{~d} s \\
& \leq \frac{1}{d} \exp (-M t)\left(\left\|v_{1}^{0}(\omega)\right\|_{2}^{2}+\left\|v_{2}^{0}(\omega)\right\|_{2}^{2}\right)+\hat{C} \\
& \quad+\tilde{C} \int_{0}^{t} \exp (M(s-t))\left(\left\|z_{2}\left(\theta_{s} \omega\right)\right\|_{2}^{2}+\left\|z_{1}\left(\theta_{s} \omega\right)\right\|_{p}^{p}\right) \mathrm{d} s
\end{aligned}
$$

as well as

$$
\begin{aligned}
& \left\|v_{1}\left(t, \omega, v_{1}^{0}(\omega)\right)\right\|_{2}^{2}+\left\|v_{2}\left(t, \omega, v_{2}^{0}(\omega)\right)\right\|_{2}^{2} \\
& \leq\left(\left\|v_{1}^{0}(\omega)\right\|_{2}^{2}+\left\|v_{2}^{0}(\omega)\right\|_{2}^{2}\right) \exp (-M t)+\hat{C} \\
& \quad+\tilde{C} \int_{0}^{t} \exp (M(s-t))\left(\left\|z_{2}\left(\theta_{s} \omega\right)\right\|_{2}^{2}+\left\|z_{1}\left(\theta_{s} \omega\right)\right\|_{p}^{p}\right) \mathrm{d} s .
\end{aligned}
$$

In particular, from the last estimate we obtain

$$
\begin{aligned}
& \int_{0}^{t_{\mathcal{D}}(\omega)}\left(\| v_{1}\left(s, \theta_{-t} \omega, v_{1}^{0}\left(\theta_{-t} \omega\right)\left\|_{2}^{2}+\right\| v_{2}\left(s, \theta_{-t} \omega, v_{2}^{0}\left(\theta_{-t} \omega\right)\right) \|_{2}^{2}\right) \exp (M(s-t)) \mathrm{d} s\right. \\
& \leq \int_{0}^{t_{\mathcal{D}}(\omega)}\left(\left\|v_{1}^{0}\left(\theta_{-t} \omega\right)\right\|_{2}^{2}+\left\|v_{2}^{0}\left(\theta_{-t} \omega\right)\right\|_{2}^{2}\right) \exp (-M t) \mathrm{d} s+\hat{C} \int_{0}^{t_{\mathcal{D}}(\omega)} \exp (M(s-t)) \mathrm{d} s \\
& \quad+\tilde{C} \int_{0}^{t_{\mathcal{D}}(\omega)} \int_{0}^{s} \exp (M(\tau-t))\left(\left\|z_{2}\left(\theta_{\tau-t} \omega\right)\right\|_{2}^{2}+\left\|z_{1}\left(\theta_{\tau-t} \omega\right)\right\|_{p}^{p}\right) \mathrm{d} \tau \mathrm{d} s \\
& \leq\left(\left\|v_{1}^{0}\left(\theta_{-t} \omega\right)\right\|_{2}^{2}+\left\|v_{2}^{0}\left(\theta_{-t} \omega\right)\right\|_{2}^{2}\right) \exp (-M t) t_{\mathcal{D}}(\omega)+\hat{C} \\
& \quad+\tilde{C} t_{\mathcal{D}}(\omega) \int_{0}^{t_{\mathcal{D}}(\omega)} \exp (M(\tau-t))\left(\left\|z_{2}\left(\theta_{\tau-t} \omega\right)\right\|_{2}^{2}+\left\|z_{1}\left(\theta_{\tau-t} \omega\right)\right\|_{p}^{p}\right) \mathrm{d} \tau
\end{aligned}
$$

where we have replaced $\omega$ by $\theta_{-t} \omega$ after integrating and used that $t \geq t_{\mathcal{D}}(\omega)$. 
Now, replacing $\omega$ by $\theta_{-t} \omega$ in (3.39), noting that $\delta \geq M$ and assuming that $t \geq t_{\mathcal{D}}(\omega)$, we compute

$$
\begin{aligned}
&\left\|\nabla v_{2}^{1}\left(t, \theta_{-t} \omega, 0\right)\right\|_{2}^{2} \\
& \leq \frac{C_{1}}{\delta}+C_{2} \int_{0}^{t}\left[\left\|v_{2}^{1}\left(s, \theta_{-t} \omega, 0\right)\right\|_{2}^{2}+\left\|v_{1}\left(s, \theta_{-t} \omega, v_{1}^{0}\left(\theta_{-t} \omega\right)\right)\right\|_{2}^{2}+\left\|z_{1}\left(\theta_{s-t} \omega\right)\right\|_{2}^{2}\right. \\
&\left.+\left\|\nabla v_{1}\left(s, \theta_{-t} \omega, v_{1}^{0}\left(\theta_{-t} \omega\right)\right)\right\|_{2}^{2}+\left\|\nabla z_{1}\left(\theta_{s-t} \omega\right)\right\|_{2}^{2}\right] \exp ((s-t) M) \mathrm{d} s \\
& \leq C_{1}+C_{2} \int_{0}^{t_{\mathcal{D}}(\omega)}\left[\left\|v_{2}^{1}\left(s, \theta_{-t} \omega, 0\right)\right\|_{2}^{2}+\left\|v_{1}\left(s, \theta_{-t} \omega, v_{1}^{0}\left(\theta_{-t} \omega\right)\right)\right\|_{2}^{2}\right] \exp ((s-t) M) \mathrm{d} s \\
&+C_{2} \int_{t_{\mathcal{D}}(\omega)}^{t}\left[\left\|v_{2}^{1}\left(s, \theta_{-t} \omega, 0\right)\right\|_{2}^{2}+\left\|v_{1}\left(s, \theta_{-t} \omega, v_{1}^{0}\left(\theta_{-t} \omega\right)\right)\right\|_{2}^{2}\right] \exp ((s-t) M) \mathrm{d} s \\
&+C_{3} \exp (-M t)\left(\left\|v_{1}^{0}\left(\theta_{-t} \omega\right)\right\|_{2}^{2}+\left\|v_{2}^{0}\left(\theta_{-t} \omega\right)\right\|_{2}^{2}\right)+C_{4} \int_{0}^{t} \exp (M(s-t)) \\
& \times\left(\left\|z_{2}\left(\theta_{s-t} \omega\right)\right\|_{2}^{2}+\left\|z_{1}\left(\theta_{s-t} \omega\right)\right\|_{p}^{p}+\left\|z_{1}\left(\theta_{s-t} \omega\right)\right\|_{2}^{2}+\left\|\nabla z_{1}\left(\theta_{s-t} \omega\right)\right\|_{2}^{2}\right) \mathrm{d} s \\
& \leq C_{1}+C_{2}\left(\left\|v_{1}^{0}(\theta-t \omega)\right\|_{2}^{2}+\left\|v_{2}^{0}\left(\theta_{-t} \omega\right)\right\|_{2}^{2}\right) \exp (-M t) t_{\mathcal{D}}(\omega) \\
&+C_{5} t_{\mathcal{D}}(\omega) \int_{0}^{t_{\mathcal{D}}(\omega)} \exp (M(\tau-t))\left(\left\|z_{2}\left(\theta_{\tau-t} \omega\right)\right\|_{2}^{2}+\left\|z_{1}\left(\theta_{\tau-t} \omega\right)\right\|_{p}^{p}\right) \mathrm{d} \tau \\
&+C_{2} \int_{t_{\mathcal{D}}(\omega)}^{t} \rho(\omega) \exp ((s-t) M) \mathrm{d} s \\
&+C_{3} \exp (-M t)\left(\left\|v_{1}^{0}\left(\theta_{-t} \omega\right)\right\|_{2}^{2}+\left\|v_{2}^{0}\left(\theta_{-t} \omega\right)\right\|_{2}^{2}\right) \\
&+C_{4} \int_{-\infty}^{0} \exp (M s)\left(\left\|z_{2}\left(\theta_{s} \omega\right)\right\|_{2}^{2}+\left\|z_{1}\left(\theta_{s} \omega\right)\right\|_{p}^{p}+\left\|z_{1}\left(\theta_{s} \omega\right)\right\|_{2}^{2}+\left\|\nabla z_{1}\left(\theta_{s} \omega\right)\right\|_{2}^{2}\right) \mathrm{d} s \\
& \leq C_{1}+C_{2}\left(t_{\mathcal{D}}(\omega)\right)\left(\left\|v_{1}^{0}(\theta-t \omega)\right\|_{2}^{2}+\left\|v_{2}^{0}\left(\theta_{-t} \omega\right)\right\|_{2}^{2}\right) \exp (-M t)+C_{3} \rho(\omega) \\
&+C_{4}\left(t_{\mathcal{D}}(\omega)\right) \int_{-\infty}^{0} \exp (M s) \\
& \times\left(\left\|z_{2}\left(\theta_{s} \omega\right)\right\|_{2}^{2}+\left\|z_{1}\left(\theta_{s} \omega\right)\right\|_{p}^{p}+\left\|z_{1}\left(\theta_{s} \omega\right)\right\|_{2}^{2}+\left\|\nabla z_{1}\left(\theta_{s} \omega\right)\right\|_{2}^{2}\right) \mathrm{d} s
\end{aligned}
$$

where we have used (3.41) in the second inequality and (3.42) in the third inequality. Furthermore, we made use of the absorption property in the third inequality. Finally, since $\left\|z_{2}\left(\theta_{s} \omega\right)\right\|_{2}^{2},\left\|z_{1}\left(\theta_{s} \omega\right)\right\|_{p}^{p},\left\|z_{1}\left(\theta_{s} \omega\right)\right\|_{2}^{2},\left\|\nabla z_{1}\left(\theta_{s} \omega\right)\right\|_{2}^{2}$ (see Lemma 3.17 and Remark 3.18) and $\left\|v_{1}^{0}\left(\theta_{-t} \omega\right)\right\|_{2}^{2},\left\|v_{2}^{0}\left(\theta_{-t} \omega\right)\right\|_{2}^{2}$ (by assumption) are tempered random variables, we can combine the right hand side into one tempered random variable $\rho_{2}(\omega)$ and this concludes the proof.

Theorem 3.26. Let Assumptions 2.1 and 2.2 hold. The random dynamical system defined in (3.11) has a unique $\mathcal{T}$-random attractor $\mathcal{A}$.

Proof. By the previous lemmas there exist a compact absorbing set given by (3.28) in $\mathcal{T}$ for the $\operatorname{RDS} \varphi$. Thus Theorem 3.11 guarantees the existence of a unique $\mathcal{T}$-random attractor. 


\section{Applications}

\subsection{FitzHugh-Nagumo system}

Let us consider the famous stochastic FitzHugh-Nagumo system, i.e.,

$$
\begin{aligned}
& \mathrm{d} u_{1}=\left(\nu_{1} \Delta u_{1}-p(x) u_{1}-u_{1}\left(u_{1}-1\right)\left(u_{1}-\alpha_{1}\right)-u_{2}\right) \mathrm{d} t+B_{1} \mathrm{~d} W_{1}, \\
& \mathrm{~d} u_{2}=\left(\alpha_{2} u_{1}-\alpha_{3} u_{2}\right) \mathrm{d} t+B_{2} \mathrm{~d} W_{2},
\end{aligned}
$$

with $D=[0,1]$ and $\alpha_{j} \in \mathbb{R}$ for $j \in\{1,2,3\}$ are fixed parameters. We always assume that the noise terms satisfy Assumptions 2.2 and $p \in C^{2}$. Such systems have been considered under various assumptions by numerous authors, for instance see $[4,31]$ and the references specified therein. Our general assumptions are satisfied in this example as follows. Identifying the terms with the terms given in (2.1)-(2.2) we have

$$
\begin{aligned}
& h\left(x, u_{1}\right)=p(x) u_{1}+u_{1}\left(u_{1}-1\right)\left(u_{1}-\alpha_{1}\right), \quad f\left(x, u_{1}, u_{2}\right)=u_{2}, \\
& \sigma(x) u_{2}=\alpha_{3} u_{2}, \quad g\left(x, u_{1}\right)=-\alpha_{2} u_{1} .
\end{aligned}
$$

We have $\sigma(x)=\alpha_{3}$ and $\left|f\left(x, u_{1}, u_{2}\right)\right|=\left|u_{2}\right|$, i.e., (2.7) and (2.6) are fulfilled. Furthermore, $\left|\partial_{u} g\left(x, u_{1}\right)\right|=\left|\alpha_{2}\right|$ and $\left|\partial_{x_{i}} g\left(x, u_{1}\right)\right|=0$ for $i=1, \ldots, n$, hence (2.8) is satisfied. Finally, as a polynomial with odd degree and negative coefficient for the highest degree, $h$ fulfils (2.5). Thus the analysis above guarantees the existence of global mild solutions and the existence of a random pullback attractor for the stochastic FitzHugh-Nagumo system.

\subsection{The driven cubic-quintic Allen-Cahn model}

The cubic-quintic Allen-Cahn (or real Ginzburg-Landau) equation is given by

$$
\partial_{t} u=\Delta u+p_{1} u+u^{3}-u^{5}, \quad u=u(x, t),
$$

where $(x, t) \in D \times[0, T), p_{1} \in \mathbb{R}$, is a fixed parameter and we will take $D$ as a bounded open domain with regular boundary. The cubic-quintic polynomial non-linearity frequently occurs in the modelling of Euler buckling [30], as a re-stabilization mechanism in paradigmatic models for fluid dynamics [21], in normal form theory and travelling wave dynamics $[13,16]$, as well as a test problem for deterministic [17] and stochastic numerical continuation [18]. If we want to allow for time-dependent slowly-varying forcing on $u$ and sufficiently regular additive noise, then it is actually very natural to extend the model (4.2) to

$$
\begin{aligned}
& \mathrm{d} u_{1}=\left(\Delta u_{1}+p_{1} u_{1}+u_{1}^{3}-u_{1}^{5}-u_{2}\right) \mathrm{d} t+B_{1} \mathrm{~d} W_{1}, \\
& \mathrm{~d} u_{2}=\varepsilon\left(p_{2} u_{2}-q_{2} u_{1}\right) \mathrm{d} t+B_{2} \mathrm{~d} W_{2},
\end{aligned}
$$

where $p_{2}, q_{2}, 0<\varepsilon \ll 1$ are parameters. One easily checks again that (4.3) fits our general framework as $h\left(x, u_{1}\right)=-p_{1} u_{1}-u_{1}^{3}+u_{1}^{5}$ satisfies the crucial dissipation assumption (2.5).

\section{Acknowledgements}

Open Access funding provided by Projekt DEAL. 
Open Access. This article is licensed under a Creative Commons Attribution 4.0 International License, which permits use, sharing, adaptation, distribution and reproduction in any medium or format, as long as you give appropriate credit to the original author(s) and the source, provide a link to the Creative Commons licence, and indicate if changes were made. The images or other third party material in this article are included in the article's Creative Commons licence, unless indicated otherwise in a credit line to the material. If material is not included in the article's Creative Commons licence and your intended use is not permitted by statutory regulation or exceeds the permitted use, you will need to obtain permission directly from the copyright holder. To view a copy of this licence, visit http://creativecommons. org/licenses/by/4.0/.

Publisher's Note Springer Nature remains neutral with regard to jurisdictional claims in published maps and institutional affiliations.

\section{References}

[1] Adili, A., Wang, B.: Random attractors for non-autonomous stochastic FitzHugh-Nagumo systems with multiplicative noise. In: Discrete and Continuous Dynamical Systems SI (2013)

[2] Adili, A., Wang, B.: Random attractors for stochastic FitzHugh-Nagumo systems driven by determinisits non-autonomous forcing. Discrete Contin. Dyn. Syst. Ser. B 18(3), 643-666 (2013)

[3] Arnold, L.: Random Dynamical Systems. Springer, Berlin (2013)

[4] Bonaccorsi, S., Mastrogiacomo, E.: Analysis of the stochastic FitzHugh-Nagumo system. Infin. Dimens. Anal. Quantum Probab. Relat. Top. 11(03), 427-446 (2008)

[5] Caraballo, T., Langa, J.A., Robinson, J.C.: Stability and random attractors for a reaction-diffusion equation with multiplicative noise. Discrete Contin. Dyn. Syst. 6(4), 875-892 (2000)

[6] Chepyzhov, V.V., Vishik, M.I.: Trajectory attractors for reaction-diffusion systems. Topol. Methods Nonlinear Anal. 7(1), 49-76 (1996)

[7] Chueshov, I., Schmalfuss, B.: Master-slave synchronization and invariant manifolds for coupled stochastic systems. J. Math. Phys. 51(10), 102702 (2010)

[8] Crauel, H., Debussche, A., Flandoli, F.: Random attractors. J. Dyn. Differ. Equ. 9(2), 307-341 (1997)

[9] Crauel, H., Flandoli, F.: Attractors for random dynamical systems. Probab. Theory Relat. Fields 100(3), 365-393 (1994)

[10] Da Prato, G.: Kolmogorov Equations for Stochastic PDEs. Birkhäuser, Basel (2012)

[11] Da Prato, G., Zabczyk, J.: Stochastic Equations in Infinite Dimensions. Cambridge University Press, Cambridge (1992) 
[12] Debussche, A.: Hausdorff dimension of a random invariant set. J. Math. Pures Appl. 77(10), 967-988 (1998)

[13] Deissler, R., Brand, H.: Periodic, quasiperiodic, and chaotic localized solutions of the quintic complex Ginzburg-Landau equation. Phys. Rev. Lett. 72(4), 478-481 (1994)

[14] Flandoli, F., Schmalfuss, B.: Random attractors for the 3D stochastic NavierStokes equation with multiplicative white noise. Stoch. Int. J. Probab. Stoch. Process. 59(1-2), 21-45 (1996)

[15] Gess, B., Liu, W., Röckner, M.: Random attractors for a class of stochastic partial differential equations driven by general additive noise. J. Differ. Equ. 251(4-5), 1225-1253 (2011)

[16] Kapitula, T., Sandstede, B.: Instability mechanism for bright solitary-wave solutions to the cubic-quintic Ginzburg-Landau equation. JOSA B 15(11), 27572762 (1998)

[17] Kuehn, C.: Efficient gluing of numerical continuation and a multiple solution method for elliptic PDEs. Appl. Math. Comput. 266, 656-674 (2015)

[18] Kuehn, C.: Numerical continuation and SPDE stability for the 2d cubic-quintic Allen-Cahn equation. SIAM/ASA J. Uncertain. Quantif. 3(1), 762-789 (2015)

[19] Li, Y., Yin, J.: A modified proof of pullback attractors in a sobolev space for stochastic Fitzhugh-Nagumo equations. Discrete Contin. Dyn. Syst. Ser. B 21(4), 1203-1223 (2016)

[20] Marion, M.: Finite-dimensional attractors associated with partly dissipative reaction-diffusion systems. SIAM J. Math. Anal. 20(4), 816-844 (1989)

[21] Morgan, D., Dawes, J.: The Swift-Hohenberg equation with a nonlocal nonlinearity. Physica D 270, 60-80 (2014)

[22] Nagel, R.: Towards a "matrix theory" for unbounded operator matrices. Math. Z. 201(1), 57-68 (1989)

[23] Pazy, A.: Semigroups of Linear Operators and Applications to Partial Differential Equations. Springer, Berlin (2012)

[24] Sauer, M., Stannat, W.: Analysis and approximation of stochastic nerve axon equations. Math. Comput. 85(301), 2457-2481 (2016)

[25] Schmalfuss, B.: Backward cocycles and attractors of stochastic differential equations. In: International seminar on applied mathematics-nonlinear dynamics: attractor approximation and global behaviour, pp 185-192 (1992)

[26] Schmalfuss, B.: Attractors for the non-autonomous dynamical systems. In: Equadiff 99, vol 2, pp 684-689. World Scientific (2000)

[27] Sell, G.R., You, Y.: Dynamics of Evolutionary Equations. Springer, Berlin (2002)

[28] Temam, R.: Infinite-Dimensional Dynamical Systems in Mechanics and Physics. Springer, Berlin (2012) 
[29] Van Neerven, J.: Stochastic Evolution Equations. ISEM Lecture Notes. Cambridge University of Press, Cambridge (2008)

[30] Venkadesan, M., Guckenheimer, J., Valero-Cuevas, F.: Manipulating the edge of instability. J. Biomech. 40, 1653-1661 (2007)

[31] Wang, B.: Attractors for reaction-diffusion equations in unbounded domains. Physica D 128(1), 41-52 (1999)

[32] Wang, B.: Pullback attractors for the non-autonomous FitzHugh-Nagumo system on unbounded domains. Nonlinear Anal. Theor. 70(11), 3799-3815 (2009)

[33] Wang, B.: Random attractors for the stochastic FitzHugh-Nagumo system on unbounded domains. Nonlinear Anal. Theor. 71(7-8), 2811-2828 (2009)

[34] Zeidler, E.: Nonlinear Functional Analysis and Its Applications: Part 2 B: Nonlinear Monotone Operators. Springer, Berlin (1989)

[35] Zhou, S., Wang, Z.: Finite fractal dimensions of random attractors for stochastic FitzHugh-Nagumo system with multiplicative white noise. J. Math. Anal. Appl. 441(2), 648-667 (2016)

Christian Kuehn, Alexandra Neamţu and Anne Pein

Faculty of Mathematics

Technical University of Munich

Boltzmannstr. 3

85748 Garching bei München

Germany

e-mail: pein@ma.tum.de

Christian Kuehn

e-mail: ckuehn@ma.tum.de

Alexandra Neamţu

e-mail: neamtu@ma.tum.de

Received: 22 July 2019.

Accepted: 11 May 2020. 